\title{
Moments of nucleon isovector structure functions in 2+1+1-flavor QCD
}

\author{
Santanu Mondal@, ${ }^{1, *}$ Rajan Gupta๑, ${ }^{1, \dagger}$ Sungwoo Park $\odot,{ }^{1, ٪}$ Boram Yoon $\odot,{ }^{2, \S}$ \\ Tanmoy Bhattacharya $\odot^{1, \|}$ and Huey-Wen Lin $\oplus^{3,4, \uparrow}$ \\ (Precision Neutron Decay Matrix Elements (PNDME) Collaboration) \\ ${ }^{1}$ Los Alamos National Laboratory, Theoretical Division T-2, Los Alamos, New Mexico 87545, USA \\ ${ }^{2}$ Los Alamos National Laboratory, Computer Computational and Statistical Sciences, \\ CCS-7, Los Alamos, New Mexico 87545, USA \\ ${ }^{3}$ Department of Physics and Astronomy, Michigan State University, Michigan 48824, USA \\ ${ }^{4}$ Department of Computational Mathematics, Science and Engineering, Michigan State University, \\ East Lansing, Michigan 48824, USA
}

(Received 4 June 2020; accepted 3 August 2020; published 22 September 2020)

\begin{abstract}
We present results on the isovector momentum fraction, $\langle x\rangle_{u-d}$, helicity moment, $\langle x\rangle_{\Delta u-\Delta d}$, and the transversity moment, $\langle x\rangle_{\delta u-\delta d}$, of the nucleon obtained using nine ensembles of gauge configurations generated by the MILC Collaboration using $2+1+1$-flavors of dynamical highly improved staggered quarks. The correlation functions are calculated using the Wilson-Clover action, and the renormalization of the three operators is carried out nonperturbatively on the lattice in the $\mathrm{RI}^{\prime}$-MOM scheme. The data have been collected at lattice spacings $a \approx 0.15,0.12,0.09$, and $0.06 \mathrm{fm}$ and $M_{\pi} \approx 310,220$, and $135 \mathrm{MeV}$, which are used to obtain the physical values using a simultaneous chiral-continuum-finite-volume fit. The final results, in the $\overline{\mathrm{MS}}$ scheme at $2 \mathrm{GeV}$, are $\langle x\rangle_{u-d}=0.173(14)(07),\langle x\rangle_{\Delta u-\Delta d}=0.213(15)(22)$, and $\langle x\rangle_{\delta u-\delta d}=0.208(19)(24)$, where the first error is the overall analysis uncertainty and the second is an additional systematic uncertainty due to possible residual excited-state contributions. These results are consistent with other recent lattice calculations and phenomenological global fit values.
\end{abstract}

DOI: $10.1103 /$ PhysRevD.102.054512

\section{INTRODUCTION}

The elucidation of the hadron structure in terms of quarks and gluons is evolving from determining the charges and form factors of nucleons to including more complex quantities such as parton distribution functions (PDFs) [1], transverse momentum dependent PDFs (TMDs) [2], and generalized parton distributions (GPDs) [3] as experiments become more precise [4,5]. These distributions are not measured directly in experiments, and phenomenological analyses including different theoretical inputs are needed to

\footnotetext{
*santanu@lanl.gov

†rajan@lanl.gov

*sungwoo@lanl.gov

§oram@lanl.gov

"tanmoy@lanl.gov

"hwlin@pa.msu.edu
}

Published by the American Physical Society under the terms of the Creative Commons Attribution 4.0 International license. Further distribution of this work must maintain attribution to the author(s) and the published article's title, journal citation, and DOI. Funded by SCOAP ${ }^{3}$. extract them from experimental data. Input from lattice QCD is beginning to play an increasingly larger role in such analyses [6]. In cases where both lattice results and phenomenological analyses of experimental data (global fits) exist, one can compare them to validate the control over systematics in the lattice calculations and, on the other hand, provide a check on the phenomenological process used to extract these observables from experimental data. In other cases, lattice results are predictions. The list of quantities for which good agreement between lattice calculations and experimental results, and their precision, has grown very significantly as discussed in the recent Flavour Lattice Averaging Group (FLAG) 2019 report [7]. While steady progress has been made in developing the framework for calculating distribution functions using lattice QCD [8,9], even calculations of their moments have had large statistical and/or systematic uncertainties prior to 2018. This was the case even for the best studied quantity, the isovector momentum fraction $\langle x\rangle_{u-d}$ [6]. In this work, we show that the lattice data for the momentum fraction, helicity, and transversity moments are now of quality comparable to that for nucleon charges (zeroth moments). 
TABLE I. Lattice parameters, nucleon mass $M_{N}$, number of configurations analyzed, and the total number of high precision (HP) and low precision (LP) measurements made. For the $a 06 \mathrm{~m} 310 \mathrm{~W}$ ensemble, HP data were not collected; however, we note that the bias correction factor on all other eight ensembles was negligible.

\begin{tabular}{|c|c|c|c|c|c|c|c|c|c|}
\hline Ensemble ID & $a[\mathrm{fm}]$ & $M_{\pi}^{\mathrm{val}}[\mathrm{MeV}]$ & $L^{3} \times T$ & $M_{\pi}^{\mathrm{val} L}$ & $\tau / a$ & $a M_{N}$ & $N_{\text {conf }}$ & $N_{\mathrm{HP}}$ & $N_{\text {LP }}$ \\
\hline$a 15 m 310$ & $0.1510(20)$ & $320.6(4.3)$ & $16^{3} \times 48$ & 3.93 & $\{5,6,7,8,9\}$ & $0.8287(24)$ & 1917 & 7,668 & 122,688 \\
\hline$a 12 m 310$ & $0.1207(11)$ & $310.2(2.8)$ & $24^{3} \times 64$ & 4.55 & $\{8,10,12,14\}$ & $0.6660(27)$ & 1013 & 8,104 & 64,832 \\
\hline$a 12 m 220$ & $0.1184(09)$ & 227.9(1.9) & $32^{3} \times 64$ & 4.38 & $\{8,10,12,14\}$ & $0.6289(26)$ & 1156 & 4,624 & 73,984 \\
\hline$a 12 m 220 L$ & $0.1189(09)$ & 227.6(1.7) & $40^{3} \times 64$ & 5.49 & $\{8,10,12,14\}$ & $0.6125(21)$ & 1000 & 4,000 & 128,000 \\
\hline$a 09 m 310$ & $0.0888(08)$ & $313.0(2.8)$ & $32^{3} \times 96$ & 4.51 & $\{10,12,14,16\}$ & $0.4951(13)$ & 2263 & 9,052 & 144,832 \\
\hline$a 09 m 220$ & $0.0872(07)$ & $225.9(1.8)$ & $48^{3} \times 96$ & 4.79 & $\{10,12,14,16\}$ & $0.4496(18)$ & 960 & 7,668 & 122,688 \\
\hline$a 09 m 130$ & $0.0871(06)$ & 138.1(1.0) & $64^{3} \times 96$ & 3.90 & $\{10,12,14,16\}$ & $0.4204(23)$ & 1041 & 8,328 & 99, 936 \\
\hline$a 06 m 310 W$ & $0.0582(04)$ & $319.6(2.2)$ & $48^{3} \times 144$ & 4.52 & $\{18,20,22,24\}$ & $0.3304(23)$ & 500 & $\ldots$ & 66,000 \\
\hline$a 06 m 135$ & $0.0570(01)$ & $135.6(1.4)$ & $96^{3} \times 192$ & 3.7 & $\{16,18,20,22\}$ & $0.2704(32)$ & 751 & 6,008 & 48,064 \\
\hline
\end{tabular}

Together with much more precise data from the planned electron-ion collider [4] and the Large Hadron Collider, which will significantly improve the phenomenological global fits, we anticipate steady progress toward a detailed description of the hadron structure.

In this paper we present results on the three moments from high statistics calculations done on nine ensembles generated using $2+1+1$-flavors of highly improved staggered quarks (HISQ) [10] by the MILC collaboration [11]. The data at four values of lattice spacings $a$, three values of the pion mass, $M_{\pi}$, including two ensembles at the physical pion mass, and on a range of large physical volumes, characterized by $M_{\pi} L$, allow us to carry out a simultaneous fit in these three variables to address the associated systematics uncertainties. We also investigate the dependence of the results on the spectra of possible excited states included in the fits to remove excited-state contamination (ESC), and assign a second error to account for the associated systematic uncertainty. Our final results are $\langle x\rangle_{u-d}=0.173(14)(07)$, $\langle x\rangle_{\Delta u-\Delta d}=0.213(15)(22)$, and $\langle x\rangle_{\delta u-\delta d}=0.208(19)(24)$ in the $\overline{\mathrm{MS}}$ scheme at $2 \mathrm{GeV}$. On comparing these with other lattice and phenomenological global fit results in Sec. VI, we find a consistent picture emerging.

The paper is organized as follows: In Sec. II, we briefly summarize the lattice parameters and methodology. The definitions of moments and operators investigated are given in Sec. III. The two- and three-point functions calculated, and their connection to the moments, are specified in Sec. IV, and the analysis of excited state contributions to extract ground state matrix elements is presented in Sec. V. Results for the moments after the chiral-continuum-finite-volume (CCFV) extrapolation are given in Sec. VI and compared with other lattice calculations and global fits. We end with conclusions in Sec. VII. The data and fits used to remove excited-state contamination are shown in the Appendix $A$ and the results for renormalization factors, $Z_{V D, A D, T D}$, for the three operators in Appendix B.

\section{LATTICE METHODOLOGY}

The parameters of the nine HISQ ensembles are summarized in Table I. They cover a range of lattice spacings $(0.057 \leq a \leq 0.15 \mathrm{fm})$, pion masses $\left(135 \leq M_{\pi} \leq 310\right)$ $\mathrm{MeV}$, and lattice sizes $\left(3.7 \leq M_{\pi} L \leq 5.5\right)$. Most of the details of the lattice methodology, the strategies for the calculations, and the analyses are already given in Refs. [12-14]. We construct the correlation functions needed to calculate the matrix elements using Wilsonclover fermions on these HISQ ensembles. This mixedaction, clover-on-HISQ, formulation is nonunitary and can suffer from the problem of exceptional configurations at small, but a priori unknown, quark masses. We have not found evidence for such exceptional configurations on any of the nine ensembles analyzed in this work.

For the parameters used in the construction of the twoand three-point functions with Wilson-clover fermion see Table II of Ref. [14]. The Sheikholeslami-Wohlert coefficient [15] used in the clover action is fixed to its tree-level value with tadpole improvement, $c_{s w}=1 / u_{0}$, where $u_{0}$ is the fourth root of the plaquette expectation value calculated on the hypercubic (HYP) smeared [16] HISQ lattices.

The masses of light clover quarks were tuned so that the clover-on-HISQ pion masses, $M_{\pi}^{\mathrm{val}}$, match the HISQon-HISQ Goldstone ones, $M_{\pi}^{\text {sea }} \cdot M_{\pi}^{\text {val }}$ values are given in Table I. $M_{\pi}^{\text {sea }}$ values are available in Ref. [14]. All fits in $M_{\pi}^{2}$ to study the chiral behavior are made using the clover-on-HISQ $M_{\pi}^{\text {val }}$ since the correlation functions, and thus the chiral behavior of the moments, have a greater sensitivity to it. Henceforth, for brevity, we drop the superscript and denote the clover-on-HISQ pion mass as $M_{\pi}$. The number of high precision (HP) and low precision (LP) measurements made on each configuration in the truncated solver bias corrected method $[17,18]$ for a cost-effective increase in statistics are specified in Table I. 


\section{MOMENTS AND MATRIX ELEMENTS}

In this work, we calculate the first moments of spin independent (or unpolarized), $q=q_{\uparrow}+q_{\downarrow}$, helicity (or polarized), $\Delta q=q_{\uparrow}-q_{\downarrow}$, and transversity, $\delta q=q_{\top}+q_{\perp}$ distributions, defined as

$$
\begin{aligned}
\langle x\rangle_{q} & =\int_{0}^{1} x[q(x)+\bar{q}(x)] d x, \\
\langle x\rangle_{\Delta q} & =\int_{0}^{1} x[\Delta q(x)+\Delta \bar{q}(x)] d x, \\
\langle x\rangle_{\delta q} & =\int_{0}^{1} x[\delta q(x)+\delta \bar{q}(x)] d x,
\end{aligned}
$$

where $q_{\uparrow(\downarrow)}$ corresponds to quarks with helicity aligned (antialigned) with that of a longitudinally polarized target, and $q_{\top(\perp)}$ corresponds to quarks with spin aligned (antialigned) with that of a transversely polarized target.

These moments, at leading twist, can be extracted from the hadron matrix elements of one-derivative vector, axialvector, and tensor operators at zero momentum transfer. The unpolarized and polarized moments $\langle x\rangle_{q}$ and $\langle x\rangle_{\Delta q}$ of the nucleon are also obtained from phenomenological global fits while a computation of the nucleon transversity $\langle x\rangle_{\delta q}$ using lattice QCD is still a prediction due to the lack of sufficient experimental data [6].

We are interested in extracting the forward nucleon matrix elements $\langle N(p)|\mathcal{O}| N(p)\rangle$, with the nucleon initial and final 3-momenta, $\vec{p}$, taken to be zero in this work. The complete set of one-derivative vector, axial-vector, and tensor operators is the following:

$$
\begin{aligned}
& \mathcal{O}_{V^{a}}^{\mu \nu}=\bar{q} \gamma^{\{\mu} \stackrel{\leftrightarrow}{D}{ }^{\nu} \tau^{a} q, \\
& \mathcal{O}_{A^{a}}^{\mu \nu}=\bar{q} \gamma^{\{\mu} \stackrel{\leftrightarrow}{D}{ }^{\nu} \gamma^{5} \tau^{a} q, \\
& \left.\mathcal{O}_{T^{a}}^{\mu \nu \rho}=\bar{q} \sigma^{[\mu\{\nu]} \stackrel{\leftrightarrow}{D}^{\rho}\right\} \tau^{a} q,
\end{aligned}
$$

where $q=\{u, d\}$ is the isodoublet of light quarks and $\sigma^{\mu \nu}=\left(\gamma^{\mu} \gamma^{\nu}-\gamma^{\nu} \gamma^{\mu}\right) / 2$. The derivative $\stackrel{\leftrightarrow}{D}_{\nu} \equiv \frac{1}{2}\left(\vec{D}_{\nu}-\overleftarrow{D}_{\nu}\right)$ consists of four terms:

$$
\begin{aligned}
\bar{\psi}\left(\Gamma \vec{D}_{\nu}-\Gamma \overleftarrow{D}_{\nu}\right) \psi(x) \equiv & \frac{1}{2}\left[\bar{\psi}(x) \Gamma U_{\nu}(x) \psi(x+\nu)\right. \\
& -\bar{\psi}(x) \Gamma U_{\nu}^{\dagger}(x-\nu) \psi(x-\nu) \\
& +\bar{\psi}(x-\nu) \Gamma U_{\nu}(x-\nu) \psi(x) \\
& \left.-\bar{\psi}(x+\nu) \Gamma U_{\nu}^{\dagger}(x) \psi(x)\right] .
\end{aligned}
$$

Lorentz indices within \{\} in Eq. (4) are symmetrized and within [] are antisymmetrized. It is also implicit that, where relevant, the traceless part of the above operators is taken. Their renormalization is carried out nonperturbatively in the regularization independent $\mathrm{RI}^{\prime}-\mathrm{MOM}$ scheme as discussed in Appendix B. A more detailed discussion of these twist-2 operators and their renormalization can be found in Refs. $[19,20]$.

In this work, we consider only isovector quantities. These are obtained from Eq. (4) by choosing $\tau^{a}=\tau^{3}$ for the Pauli matrix. The decomposition of the matrix elements of these operators in terms of the generalized form factors at zero momentum transfer is as follows:

$$
\begin{aligned}
& \left\langle N\left(p, s^{\prime}\right)\left|\mathcal{O}_{V^{a}}^{\mu \nu}\right| N(p, s)\right\rangle=\bar{u}_{N}^{p}\left(s^{\prime}\right) A_{20}(0) \gamma^{\{\mu} p^{\nu\}} u_{N}^{p}(s), \\
& \left\langle N\left(p, s^{\prime}\right)\left|\mathcal{O}_{A^{a}}^{\mu \nu}\right| N(p, s)\right\rangle=i \bar{u}_{N}^{p}\left(s^{\prime}\right) \tilde{A}_{20}(0) \gamma^{\{\mu} p^{\nu\}} \gamma^{5} u_{N}^{p}(s),
\end{aligned}
$$

$\left\langle N\left(p, s^{\prime}\right)\left|\mathcal{O}_{T^{a}}^{\mu \nu \rho}\right| N(p, s)\right\rangle=i \bar{u}_{N}^{p}\left(s^{\prime}\right) A_{T 20}(0) \sigma^{[\mu\{\nu]} p^{\rho\}} u_{N}^{p}(s)$.

The relation between the momentum fraction, the helicity moment, and the transversity moment and the generalized form factors is $\langle x\rangle_{q}=A_{20}(0),\langle x\rangle_{\Delta q}=\tilde{A}_{20}(0)$, and $\langle x\rangle_{\delta q}=$ $A_{T 20}(0)$, respectively.

We end this discussion by mentioning that other approaches have been proposed to calculate the moments of PDFs from lattice QCD in recent years [21-23].

\section{CORRELATION FUNCTIONS AND MOMENTS}

We use the following interpolating operator $\mathcal{N}$ to create/ annihilate the nucleon state

$$
\mathcal{N}=\epsilon^{a b c}\left[q_{1}^{a T}(x) C \gamma^{5} \frac{\left(1 \pm \gamma_{4}\right)}{2} q_{2}^{b}(x)\right] q_{1}^{c}(x),
$$

where $\{a, b, c\}$ are color indices, $q_{1}, q_{2} \in\{u, d\}$, and $C=$ $\gamma_{0} \gamma_{2}$ is the charge conjugation matrix. The nonrelativistic projection $\left(1 \pm \gamma_{4}\right) / 2$ is inserted to improve the signal, with the plus and minus signs applied to the forward and backward propagation in Euclidean time, respectively [19]. At zero momentum, this operator couples only to the spin $\frac{1}{2}$ state. The zero momentum two-point and threepoint nucleon correlation functions are defined as

$$
\begin{array}{r}
\boldsymbol{C}_{\alpha \beta}^{2 \mathrm{pt}}(\tau)=\sum_{\boldsymbol{x}}\left\langle 0\left|\mathcal{N}_{\alpha}(\tau, \boldsymbol{x}) \overline{\mathcal{N}}_{\beta}(0, \boldsymbol{0})\right| 0\right\rangle, \\
\boldsymbol{C}_{\mathcal{O}, \alpha \beta}^{3 \mathrm{pt}}(\tau, t)=\sum_{\boldsymbol{x}^{\prime}, \boldsymbol{x}}\left\langle 0\left|\mathcal{N}_{\alpha}(\tau, \boldsymbol{x}) \mathcal{O}\left(t, \boldsymbol{x}^{\prime}\right) \overline{\mathcal{N}}_{\beta}(0, \boldsymbol{0})\right| 0\right\rangle,
\end{array}
$$

where $\alpha$ and $\beta$ are spin indices. The source is placed at time slice 0 , the sink is at $\tau$, and the one-derivative operators, defined in Sec. III, are inserted at time slice $t$. Data have been accumulated for the values of $\tau$ specified in Table I and in each case for all intermediate times $0 \leq t \leq \tau$. 
To isolate the various operators, projected two- and three-point functions are constructed as

$$
\begin{aligned}
& C^{2 \mathrm{pt}}=\operatorname{Tr}\left(\mathcal{P}_{2 \mathrm{pt}} \boldsymbol{C}^{2 \mathrm{pt}}\right), \\
& C_{\mathcal{O}}^{3 \mathrm{pt}}=\operatorname{Tr}\left(\mathcal{P}_{3 \mathrm{pt}} \boldsymbol{C}_{\mathcal{O}}^{3 \mathrm{pt}}\right) .
\end{aligned}
$$

The projector $\mathcal{P}_{2 \mathrm{pt}}=\frac{1}{2}\left(1+\gamma_{4}\right)$ in the nucleon correlator gives the positive parity contribution for the nucleon propagating in the forward direction. For the connected three-point contributions $\mathcal{P}_{3 \mathrm{pt}}=\frac{1}{2}\left(1+\gamma_{4}\right)\left(1+i \gamma^{5} \gamma^{3}\right)$ is used. With these spin projections, the explicit operators used to calculate the forward matrix elements are as follows:

$$
\begin{gathered}
\langle x\rangle_{u-d}: \mathcal{O}_{V^{3}}^{44}=\bar{q}\left(\gamma^{4} \stackrel{\leftrightarrow}{D}{ }^{4}-\frac{1}{3} \gamma \cdot \stackrel{\leftrightarrow}{\mathbf{D}}\right) \tau^{3} q, \\
\langle x\rangle_{\Delta u-\Delta d}: \mathcal{O}_{A^{3}}^{34}=\bar{q} \gamma^{\{3} \stackrel{\leftrightarrow}{D}{ }^{4\}} \gamma^{5} \tau^{3} q, \\
\langle x\rangle_{\delta u-\delta d}: \mathcal{O}_{T^{3}}^{124}=\bar{q} \sigma^{[1\{2] \stackrel{\leftrightarrow}{D}}{ }^{4\}} \tau^{3} q .
\end{gathered}
$$

Our goal is to obtain the matrix elements $(M E)$, of these operators within the ground state of the nucleon. These $M E$ are related to the moments as follows:

$$
\begin{gathered}
\left\langle 0\left|\mathcal{O}_{V^{3}}^{44}\right| 0\right\rangle=-M_{N}\langle x\rangle_{u-d}, \\
\left\langle 0\left|\mathcal{O}_{A^{3}}^{34}\right| 0\right\rangle=-\frac{i M_{N}}{2}\langle x\rangle_{\Delta u-\Delta d}, \\
\left\langle 0\left|\mathcal{O}_{T^{3}}^{124}\right| 0\right\rangle=-\frac{i M_{N}}{2}\langle x\rangle_{\delta u-\delta d},
\end{gathered}
$$

where $M_{N}$ is the nucleon mass. The three moments are dimensionless, and their extraction on a given ensemble does not require knowing the value of the lattice scale $a$. It enters only when performing the chiral-continuum extrapolation to the physical point as discussed in Sec. VI.

\section{CONTROLLING EXCITED STATE CONTAMINATION}

To calculate the matrix elements of the operators defined in Sec. III between ground-state nucleons, contributions of all possible excited states need to be removed. The lattice nucleon interpolating operator $\mathcal{N}$ given in Eq. (9), however, couples to the nucleon, all its excitations, and multiparticle states with the same quantum numbers. Previous lattice calculations have shown that the ESC can be large [24-26]. In our earlier works [12-14,27], we have shown that this can be controlled to within a few percent. We use the same strategy here. In particular, we use HYP smearing of the gauge links before calculating Wilson-clover quark propagators with optimized Gaussian smeared sources using the multigrid algorithm $[28,29]$. Correlation functions constructed from these smeared source propagators have smaller excited state contamination [27]. To extract the ground state matrix elements from these, we fit the threepoint data at several $\tau$ values (listed in Table I) simultaneously using the spectral decomposition given in Eq. (21).

Fits to the zero-momentum two-point functions, $C_{2 \mathrm{pt}}$, were carried out keeping up to four states in the spectral decomposition:

$$
C_{2 \mathrm{pt}}(\tau)=\sum_{i=0}^{3}\left|\mathcal{A}_{i}\right|^{2} e^{-M_{i} \tau}
$$

Fits are made over a range $\left\{\tau_{\min }-\tau_{\max }\right\}$ to extract $M_{i}$ and $\mathcal{A}_{i}$, the masses and the amplitudes for the creation/ annihilation of these states by the interpolating operator $\mathcal{N}$. In fits with more than two states, estimates of the amplitudes $\mathcal{A}_{i}$ and masses $M_{i}$ for $i \geq 2$ were sensitive to the choice of the starting time slice $\tau_{\min }$. We used the largest time interval allowed by statistics, i.e., by the stability of the covariance matrix. We perform two types of 4-state fits. In the fit denoted $\{4\}$, we use the empirical Bayesian technique described in Ref. [30] to stabilize the three excited-state parameters. In the second fit, denoted $\left\{4^{N \pi}\right\}$, we use as prior for $M_{1}$ either the noninteracting energy of $N(-\mathbf{1}) \pi(\mathbf{1})$ or the $N(\mathbf{0}) \pi(\mathbf{0}) \pi(\mathbf{0})$ state, which are both lower than the $M_{1}$ obtained from the $\{4\}$ fit, and roughly equal for the nine ensembles. The lower energy $N(\mathbf{- 1}) \pi(\mathbf{1})$ state has been shown to contribute in the axial channel [31], whereas for the vector channel the $N(\mathbf{0}) \pi(\mathbf{0}) \pi(\mathbf{0})$ state is expected to be the relevant one. We find that these two fits to the two-point function cannot be distinguished on the basis of the $\chi^{2} /$ dof; in fact, the full range of $M_{1}$ between the two estimates from $\{4\}$ and $\left\{4^{N \pi}\right\}$ are viable first-excited-state masses on the basis of $\chi^{2} /$ dof alone. The same is true of the values for $M_{2}$. We therefore, investigate the dependence of the results for moments on the excited-state spectra by doing the full analysis with multiple strategies as discussed below. The ground-state nucleon mass obtained from the various fits is denoted by the common symbol $M_{N} \equiv M_{0}$ and the mass gaps by $\Delta M_{i} \equiv M_{i}-M_{i-1}$.

The analysis of the zero-momentum three-point functions, $C_{\mathcal{O}}^{3 \text { pt }}$, is performed retaining up to three states $|i\rangle$ in the spectral decomposition:

$$
C_{\mathcal{O}}^{3 \mathrm{pt}}(\tau ; t)=\sum_{i, j=0}^{2}\left|\mathcal{A}_{i}\right|\left|\mathcal{A}_{j}\right|\langle i|\mathcal{O}| j\rangle e^{-M_{i} t-M_{j}(\tau-t)} .
$$

The operators, $\mathcal{O}$, are defined in Eqs. (14), (15), and (16). By fixing the momentum at the sink to zero and inserting the operator at zero momentum transfer we get the forward matrix element. The practical challenge discussed above is determining the relevant $M_{1}$ and $M_{2}$ to use and, failing that, 
TABLE II. Our best estimates of the unrenormalized moments from the two fit strategies, $\left\{4,3^{*}\right\}$ and $\left\{4,2^{\text {free }}\right\}$, used to analyze the two- and three-point functions. The second column gives the values of $\tau$ used in the fits, and the third column lists $t_{\text {skip }}=\{i, j\}$, the number of time slices from the source and sink omitted for each $\tau$ for the two fit types to the three-point functions. For each fit-type we give the result for the ground state matrix element, $M E$, the moment $\langle x\rangle$ obtained from it using Eqs. (17)-(19), and the $\chi^{2} /$ dof of the fit to the three-point function. In two cases, the values of $\tau / a$ included are different: the $*$ in the second column denotes $\tau / a=\{22,20,18\}$ and $\dagger$ denotes $\tau / a=\{9,8\}$ were used for the $\left\{4,2^{\text {free }}\right\}$ fits.

\begin{tabular}{|c|c|c|c|c|c|c|c|c|c|}
\hline \multirow[b]{2}{*}{ Ensemble } & \multirow[b]{2}{*}{$\tau / a$} & \multirow[b]{2}{*}{$t_{\text {skip }}$} & \multirow[b]{2}{*}{ Observable } & \multicolumn{3}{|c|}{$4,3^{*}$} & \multicolumn{3}{|c|}{$4,2^{\text {free }}$} \\
\hline & & & & $M E$ & $\langle x\rangle$ & $\chi^{2} /$ dof & $M E$ & $\langle x\rangle$ & $\chi^{2} /$ dof \\
\hline$a 06 m 135$ & $\{22,20,18\}$ & $\{4,5\}$ & $\langle x\rangle_{u-d}$ & $-0.042(4)$ & $0.155(14)$ & 0.87 & $-0.045(5)$ & $0.167(18)$ & 0.99 \\
\hline$a 06 m 135$ & $\{22,20,18\}$ & $\{4,5\}$ & $\langle x\rangle_{\Delta u-\Delta d}$ & $-0.026(2)$ & $0.191(12)$ & 1.00 & $-0.027(3)$ & $0.198(22)$ & 1.13 \\
\hline$a 06 m 135$ & $\{22,20,18\}$ & $\{4,5\}$ & $\langle x\rangle_{\delta u-\delta d}$ & $-0.025(2)$ & $0.185(16)$ & 1.32 & $-0.027(3)$ & $0.202(23)$ & 1.31 \\
\hline$a 06 m 310 \mathrm{~W}$ & $\{24,22,20\}^{*}$ & $\{6,6\}$ & $\langle x\rangle_{u-d}$ & $-0.056(4)$ & $0.170(13)$ & 1.02 & $-0.063(3)$ & $0.193(8)$ & 1.10 \\
\hline$a 06 m 310 \mathrm{~W}$ & $\{24,22,20\}$ & $\{6,6\}$ & $\langle x\rangle_{\Delta u-\Delta d}$ & $-0.037(2)$ & $0.223(15)$ & 1.00 & $-0.038(1)$ & $0.231(7)$ & 1.33 \\
\hline$a 06 m 310 \mathrm{~W}$ & $\{24,22,20\}$ & $\{6,6\}$ & $\langle x\rangle_{\delta u-\delta d}$ & $-0.035(3)$ & $0.213(18)$ & 0.80 & $-0.037(1)$ & $0.227(8)$ & 0.83 \\
\hline$a 09 m 130$ & $\{16,14,12\}$ & $\{3,3\}$ & $\langle x\rangle_{u-d}$ & $-0.074(3)$ & $0.177(8)$ & 0.93 & $-0.077(4)$ & $0.184(9)$ & 0.88 \\
\hline$a 09 m 130$ & $\{16,14,12\}$ & $\{3,3\}$ & $\langle x\rangle_{\Delta u-\Delta d}$ & $-0.046(2)$ & $0.218(7)$ & 1.30 & $-0.048(1)$ & $0.228(5)$ & 1.33 \\
\hline$a 09 m 130$ & $\{16,14,12\}$ & $\{3,3\}$ & $\langle x\rangle_{\delta u-\delta d}$ & $-0.045(2)$ & $0.212(11)$ & 1.30 & $-0.047(3)$ & $0.225(14)$ & 1.41 \\
\hline$a 09 m 220$ & $\{16,14,12\}$ & $\{3,3\}$ & $\langle x\rangle_{u-d}$ & $-0.082(3)$ & $0.184(5)$ & 0.89 & $-0.086(2)$ & $0.191(4)$ & 0.78 \\
\hline$a 09 m 220$ & $\{16,14,12\}$ & $\{3,3\}$ & $\langle x\rangle_{\Delta u-\Delta d}$ & $-0.051(1)$ & $0.227(4)$ & 0.92 & $-0.053(1)$ & $0.235(3)$ & 0.60 \\
\hline$a 09 m 220$ & $\{16,14,12\}$ & $\{3,3\}$ & $\langle x\rangle_{\delta u-\delta d}$ & $-0.053(1)$ & $0.234(6)$ & 1.29 & $-0.055(1)$ & $0.243(4)$ & 1.26 \\
\hline$a 09 m 310$ & $\{16,14,12\}$ & $\{3,3\}$ & $\langle x\rangle_{u-d}$ & $-0.097(2)$ & $0.196(4)$ & 1.25 & $-0.094(2)$ & $0.190(5)$ & 1.16 \\
\hline$a 09 m 310$ & $\{16,14,12\}$ & $\{3,3\}$ & $\langle x\rangle_{\Delta u-\Delta d}$ & $-0.058(1)$ & $0.233(3)$ & 1.24 & $-0.059(1)$ & $0.238(3)$ & 1.25 \\
\hline$a 09 m 310$ & $\{16,14,12\}$ & $\{3,3\}$ & $\langle x\rangle_{\delta u-\delta d}$ & $-0.059(1)$ & $0.239(4)$ & 0.78 & $-0.060(1)$ & $0.241(4)$ & 0.79 \\
\hline$a 12 m 220$ & $\{14,12,10\}$ & $\{3,3\}$ & $\langle x\rangle_{u-d}$ & $-0.125(5)$ & $0.199(8)$ & 1.32 & $-0.130(5)$ & $0.207(8)$ & 1.24 \\
\hline$a 12 m 220$ & $\{14,12,10\}$ & $\{3,3\}$ & $\langle x\rangle_{\Delta u-\Delta d}$ & $-0.074(3)$ & $0.234(9)$ & 0.92 & $-0.077(2)$ & $0.245(6)$ & 0.87 \\
\hline$a 12 m 220$ & $\{14,12,10\}$ & $\{3,3\}$ & $\langle x\rangle_{\delta u-\delta d}$ & $-0.077(4)$ & $0.246(11)$ & 1.24 & $-0.080(6)$ & $0.254(17)$ & 1.20 \\
\hline$a 12 m 220 L$ & $\{14,12,10\}$ & $\{3,2\}$ & $\langle x\rangle_{u-d}$ & $-0.117(6)$ & $0.191(9)$ & 1.44 & $-0.120(4)$ & $0.196(7)$ & 1.35 \\
\hline$a 12 m 220 L$ & $\{14,12,10\}$ & $\{3,3\}$ & $\langle x\rangle_{\Delta u-\Delta d}$ & $-0.073(2)$ & $0.240(7)$ & 1.33 & $-0.074(4)$ & $0.241(14)$ & 1.43 \\
\hline$a 12 m 220 L$ & $\{14,12,10\}$ & $\{3,3\}$ & $\langle x\rangle_{\delta u-\delta d}$ & $-0.073(3)$ & $0.237(10)$ & 1.25 & $-0.075(4)$ & $0.244(14)$ & 1.28 \\
\hline$a 12 m 310$ & $\{14,12,10\}$ & $\{3,3\}$ & $\langle x\rangle_{u-d}$ & $-0.130(8)$ & $0.195(11)$ & 1.66 & $-0.137(5)$ & $0.206(8)$ & 1.54 \\
\hline$a 12 m 310$ & $\{14,12,10\}$ & $\{3,3\}$ & $\langle x\rangle_{\Delta u-\Delta d}$ & $-0.079(5)$ & $0.238(16)$ & 0.76 & $-0.083(4)$ & $0.250(13)$ & 0.77 \\
\hline$a 12 m 310$ & $\{14,12,10\}$ & $\{3,3\}$ & $\langle x\rangle_{\delta u-\delta d}$ & $-0.084(6)$ & $0.251(16)$ & 0.69 & $-0.087(3)$ & $0.261(9)$ & 0.66 \\
\hline$a 15 m 310$ & $\{9,8,7\} \dagger$ & $\{2,3\}$ & $\langle x\rangle_{u-d}$ & $-0.177(5)$ & $0.214(6)$ & 1.94 & $-0.191(3)$ & $0.231(3)$ & 1.90 \\
\hline$a 15 m 310$ & $\{9,8,7\}$ & $\{2,2\}$ & $\langle x\rangle_{\Delta u-\Delta d}$ & $-0.110(3)$ & $0.266(7)$ & 0.76 & $-0.111(3)$ & $0.267(7)$ & 0.69 \\
\hline$a 15 m 310$ & $\{9,8\}$ & $\{2,2\}$ & $\langle x\rangle_{\delta u-\delta d}$ & $-0.122(5)$ & $0.293(12)$ & 0.66 & $-0.119(4)$ & $0.286(9)$ & 0.98 \\
\hline
\end{tabular}

to investigate the sensitivity of $\langle 0|\mathcal{O}| 0\rangle$ to possible values of $M_{1}$ and $M_{2}$ and including that variation as a systematic uncertainty.

For all the strategies used to determine $M_{1}$ and $M_{2}$, we extract the desired ground state matrix element $\langle 0|\mathcal{O}| 0\rangle$ by fitting the three-point correlators $C_{\mathcal{O}}^{3 \mathrm{pt}}(t ; \tau)$ for a subset of values of $t$ and $\tau$ simultaneously. This subset is chosen to reduce ESC — we select the largest values of $\tau$ and discard $t_{\text {skip }}$ number of points next to the source and sink for each $\tau$. These values of $\tau$ and of $t_{\text {skip }}$ are given in Table II.

The data for the ratio $C_{\mathcal{O}}^{3 \mathrm{pt}}(\tau ; t) / C^{2 \mathrm{pt}}(\tau)$ are shown in Figs. 5 and 6 in Appendix A for all nine ensembles. The signal in the three-point correlators decreases somewhat from momentum fraction to helicity moment to transversity moment. Nevertheless, we are able to make $3^{*}$ state (3-state with $\langle 2|\mathcal{O}| 2\rangle=0$ ) fits in all cases. The spectral decomposition predicts that the data for all three quantities is symmetric about $t=\tau / 2$; however, on some of the ensembles, and for some of the larger values of $\tau$, the data show some asymmetry, which is indicative of the size of statistical fluctuations that are present.

The fits to $C^{2 \mathrm{pt}}(\tau)$ and $C_{\mathcal{O}}^{3 \mathrm{pt}}(\tau ; t)$ are carried out within a single-elimination jackknife process, which is used to get both the central values and the errors.

We have investigated five fit types, $\{4,2\},\left\{4^{N \pi}, 2\right\}$, $\left\{4,3^{*}\right\},\left\{4^{N \pi}, 3^{*}\right\}$, and $\left\{4,2^{\text {free }}\right\}$, based on the spectral 
TABLE III. Comparison of fits using five strategies, $\{4,2\},\left\{4^{N \pi}, 2\right\},\left\{4,3^{*}\right\},\left\{4^{N \pi}, 3^{*}\right\}$, and $\left\{4,2^{\text {free }}\right\}$, for the momentum fraction $\langle x\rangle_{u-d}$ on two ensembles $a 09 m 310$ (highest statistics and $M_{\pi} \sim 310 \mathrm{MeV}$ ) and $a 06 m 135$ (physical $M_{\pi} \sim 135 \mathrm{MeV}$ ). In the $\left\{4,2^{\text {free }}\right\}$ fit, the excited state mass gap, $\Delta M_{1}$, is left as a free parameter that is determined from the fit to the three-point function. The values of $\tau / a$ and $t_{\text {skip }}$ used are the same as listed in Table II. We could not find a $\left\{4^{N \pi}, 2\right\}$ fit to the $a 06 m 135$ data that gave reasonable values.

\begin{tabular}{|c|c|c|c|c|c|c|c|c|c|}
\hline \multicolumn{10}{|c|}{$\langle x\rangle_{u-d}$} \\
\hline Ensemble & Fit-type & $a \Delta M_{1}$ & $a \Delta M_{2}$ & $\langle 0|\mathcal{O}| 0\rangle$ & $\frac{\langle 1|\mathcal{O}| 1\rangle}{\langle 0|\mathcal{O}| 0\rangle}$ & $\frac{\langle 1|\mathcal{O}| 0\rangle}{\langle 0|\mathcal{O}| 0\rangle}$ & $\frac{\langle 2|\mathcal{O}| 0\rangle}{\langle 0|\mathcal{O}| 0\rangle}$ & $\frac{\langle 2|\mathcal{O}| 1\rangle}{\langle 0|\mathcal{O}| 0\rangle}$ & $\chi^{2} /$ dof \\
\hline$a 09 m 310$ & $\{4,2\}$ & $0.434(58)$ & & $0.0982(26)$ & $4.90(3.34)$ & $0.73(7)$ & & & 1.31 \\
\hline$a 09 m 310$ & $\left\{4^{N \pi}, 2\right\}$ & $0.343(44)$ & & $0.0928(35)$ & $1.45(1.81)$ & $0.91(14)$ & & & 1.12 \\
\hline$a 09 m 310$ & $\left\{4,3^{*}\right\}$ & $0.434(58)$ & $0.697(132)$ & $0.0971(21)$ & $4.50(3.66)$ & $0.83(7)$ & $-0.27(31)$ & $-4.5(14)$ & 1.25 \\
\hline$a 09 m 310$ & $\left\{4^{N \pi}, 3^{*}\right\}$ & $0.343(44)$ & $0.555(69)$ & $0.0933(25)$ & $1.1(2.0)$ & $0.89(10)$ & $-0.01(17)$ & $2.3(4.2)$ & 1.20 \\
\hline$a 09 m 310$ & $\left\{4,2^{\text {free }}\right\}$ & $0.358(33)$ & & $0.0941(24)$ & $0.78(1.44)$ & $0.76(8)$ & & & 1.16 \\
\hline$a 06 m 135$ & $\{4,2\}$ & $0.197(37)$ & & $0.0402(56)$ & $2.6(1.5)$ & $1.12(0.31)$ & & & 0.95 \\
\hline$a 06 m 135$ & $\left\{4^{N \pi}, 2\right\}$ & $0.0846(84)$ & & $\ldots$ & $\ldots$ & $\ldots$ & & & $\cdots$ \\
\hline$a 06 m 135$ & $\left\{4,3^{*}\right\}$ & $0.197(37)$ & $0.287(49)$ & $0.0418(40)$ & $3.2(1.9)$ & $0.89(24)$ & $0.44(32)$ & $-2(5)$ & 0.87 \\
\hline$a 06 m 135$ & $\left\{4^{N \pi}, 3^{*}\right\}$ & $0.0846(84)$ & $0.201(23)$ & $0.038(15)$ & $3.4(2.8)$ & $0.11(1)$ & $1.2(4)$ & $-0.3(2.2)$ & 0.90 \\
\hline$a 06 m 135$ & $\left\{4,2^{\text {free }}\right\}$ & $0.241(49)$ & & $0.0452(47)$ & $6(6)$ & $0.99(16)$ & & & 0.99 \\
\hline
\end{tabular}

decomposition to understand and control ESC. The labels $\{m, n\}$ denote an $m$-state fit to the two-point function and an $n$-state fit to the three-point function. In the $2^{\text {free }}$-fit to the three-point function, $M_{1}$ is left as a free parameter, while a $3^{*}$-fit is a 3-state fit with $\langle 2|O| 2\rangle=0$. The results from the five strategies for the momentum fraction, $\langle x\rangle_{u-d}$, in Table III, for the helicity moment, $\langle x\rangle_{\Delta u-\Delta d}$, in Table IV, and for the transversity moment, $\langle x\rangle_{\delta u-\delta d}$, in Table V illustrate the observed behavior for the $a 09 m 310$ ensemble, which has the highest statistics, and the physical mass ensemble $a 06 m 135$ at the smallest value of $a$.

For all three observables, the five results in Tables II-VI for the ground state matrix element, $\langle 0|O| 0\rangle$, are consistent within $2 \sigma$ on the $a 09 m 310$ ensemble. On the $a 06 m 135$ ensemble, the difference in $\Delta M_{1} \equiv M_{1}-M_{0}$ between $\{4\}$ and $\left\{4^{N \pi}\right\}$ analyses becomes roughly a factor of 2 , and $\Delta M_{1}$ from the $\left\{2^{\text {free }}\right\}$ fit is larger than even the $\{4\}$ value; i.e., the $\left\{2^{\text {free }}\right\}$ fit does not prefer the small $\Delta M_{1}$ given by $\left\{4^{N \pi}\right\}$. On the other hand, the $\Delta M_{1}$ from a two-state fit is expected to be larger since it is an effective combination of the mass gaps of the full tower of excited states. Due to a small $\Delta M_{1}$, fits with the spectrum from $\left\{4^{N \pi}\right\}$ fail on $a 06 m 135$, whereas, on both ensembles, the $\left\{4,3^{*}\right\}$ and $\left\{4,2^{\text {free }}\right\}$ fits give results consistent within $2 \sigma$. The estimates from these two fit-types are given in Table II. To summarize, our overall strategy is to keep as many excited states as possible without overparametrization of the fits. We, therefore, choose, for the central values, the $\left\{4,3^{*}\right\}$ results, and to take into account the spread due to the fittype, we add a second, systematic, uncertainty to the final results in Table VII. This is taken to be the difference between the results obtained by doing the full analysis with the $\left\{4,3^{*}\right\}$ and $\left\{4,2^{\text {free }}\right\}$ strategies.

The renormalization of the matrix elements is carried out using estimates of $Z_{V D}, Z_{A D}$, and $Z_{T D}$ calculated on the lattice in the $\mathrm{RI}^{\prime}-\mathrm{MOM}$ scheme and then converted to the $\overline{\mathrm{MS}}$ scheme at $2 \mathrm{GeV}$ as described in Appendix B. The final values of $Z_{V D}, Z_{A D}$, and $Z_{T D}$ used in the analysis are given in Table IX.

TABLE IV. Comparison of fits using five strategies, $\{4,2\},\left\{4^{N \pi}, 2\right\},\left\{4,3^{*}\right\},\left\{4^{N \pi}, 3^{*}\right\}$, and $\left\{4,2^{\text {free }}\right\}$, for the helicity moment $\langle x\rangle_{\Delta u-\Delta d}$. The rest is the same as in Table III.

\begin{tabular}{|c|c|c|c|c|c|c|c|c|c|}
\hline \multicolumn{10}{|c|}{$\langle x\rangle_{\Delta u-\Delta d}$} \\
\hline Ensemble & Fit-type & $a \Delta M_{1}$ & $a \Delta M_{2}$ & $\langle 0|\mathcal{O}| 0\rangle$ & $\frac{\langle 1|\mathcal{O}| 1\rangle}{\langle 0|\mathcal{O}| 0\rangle}$ & $\frac{\langle 1|\mathcal{O}| 0\rangle}{\langle 0|\mathcal{O}| 0\rangle}$ & $\frac{\langle 2|\mathcal{O}| 0\rangle}{\langle 0|\mathcal{O}| 0\rangle}$ & $\frac{\langle 2|\mathcal{O}| 1\rangle}{\langle 0|\mathcal{O}| 0\rangle}$ & $\chi^{2} /$ dof \\
\hline$a 09 m 310$ & $\{4,2\}$ & $0.434(58)$ & & $0.115(26)$ & $2.6(2.6)$ & $0.72(5)$ & & & 1.15 \\
\hline$a 09 m 310$ & $\left\{4^{N \pi}, 2\right\}$ & $0.343(44)$ & & $0.110(33)$ & $0.33(1.5)$ & $0.85(11)$ & & & 1.43 \\
\hline$a 09 m 310$ & $\left\{4,3^{*}\right\}$ & $0.434(58)$ & $0.697(132)$ & $0.115(19)$ & $3.46(2.6)$ & $0.63(7)$ & $0.50(20)$ & $-3(12)$ & 1.24 \\
\hline$a 09 m 310$ & $\left\{4^{N \pi}, 3^{*}\right\}$ & $0.343(44)$ & $0.555(69)$ & $0.113(24)$ & $1.0(2.3)$ & $0.54(15)$ & $0.49(46)$ & $7(10)$ & 1.16 \\
\hline$a 09 m 310$ & $\left\{4,2^{\text {free }}\right\}$ & $0.539(40)$ & & $0.118(15)$ & $14(10)$ & $0.83(9)$ & & & 1.25 \\
\hline$a 06 m 135$ & $\{4,2\}$ & $0.197(37)$ & & $0.0468(61)$ & $1.07(1.09)$ & $1.07(29)$ & & & 1.29 \\
\hline$a 06 m 135$ & $\left\{4^{N \pi}, 2\right\}$ & $0.0846(84)$ & & $0.004(14)$ & $-23(110)$ & $28(115)$ & & & 0.93 \\
\hline$a 06 m 135$ & $\left\{4,3^{*}\right\}$ & $0.197(37)$ & $0.287(49)$ & $0.0517(36)$ & $4.01(1.84)$ & $0.45(21)$ & $1.28(26)$ & $-7(6)$ & 1.00 \\
\hline$a 06 m 135$ & $\left\{4^{N \pi}, 3^{*}\right\}$ & $0.0846(84)$ & $0.201(23)$ & $0.075(21)$ & $6(3)$ & $-1.3(7)$ & $1.6(3)$ & $-3.4(1.5)$ & 1.06 \\
\hline$a 06 m 135$ & $\left\{4,2^{\text {free }}\right\}$ & $0.260(67)$ & & $0.0535(60)$ & $5(7)$ & $0.98(14)$ & & & 1.18 \\
\hline
\end{tabular}


TABLE V. Comparison of fits using five strategies, $\{4,2\},\left\{4^{N \pi}, 2\right\},\left\{4,3^{*}\right\},\left\{4^{N \pi}, 3^{*}\right\}$, and $\left\{4,2^{\text {free }}\right\}$, for the transversity moment $\langle x\rangle_{\delta u-\delta d}$. The rest is the same as in Table III.

\begin{tabular}{|c|c|c|c|c|c|c|c|c|c|}
\hline \multicolumn{10}{|c|}{$\langle x\rangle_{\delta u-\delta d}$} \\
\hline Ensemble & Fit-type & $a \Delta M_{1}$ & $a \Delta M_{2}$ & $\langle 0|\mathcal{O}| 0\rangle$ & $\frac{\langle 1|\mathcal{O}| 1\rangle}{\langle 0|\mathcal{O}| 0\rangle}$ & $\frac{\langle 1|\mathcal{O}| 0\rangle}{\langle 0|\mathcal{O}| 0\rangle}$ & $\frac{\langle 2|\mathcal{O}| 0\rangle}{\langle 0|\mathcal{O}| 0\rangle}$ & $\frac{\langle 2|\mathcal{O}| 1\rangle}{\langle 0|\mathcal{O}| 0\rangle}$ & $\chi^{2} / \mathrm{dof}$ \\
\hline$a 09 m 310$ & $\{4,2\}$ & $0.434(58)$ & & $0.117(36)$ & $2.4(3.1)$ & $0.92(10)$ & & & 0.84 \\
\hline$a 09 m 310$ & $\left\{4^{N \pi}, 2\right\}$ & $0.343(44)$ & & $0.109(49)$ & $-0.83(1.9)$ & $1.17(19)$ & & & 1.45 \\
\hline$a 09 m 310$ & $\left\{4,3^{*}\right\}$ & $0.434(58)$ & $0.697(132)$ & $0.118(24)$ & $1.3(3.0)$ & $0.84(8)$ & $0.04(33)$ & $18(15)$ & 0.78 \\
\hline$a 09 m 310$ & $\left\{4^{N \pi}, 3^{*}\right\}$ & $0.343(44)$ & $0.555(69)$ & $0.115(27)$ & $-0.8(1.8)$ & $0.82(10)$ & $0.28(19)$ & $10(6)$ & 0.77 \\
\hline$a 09 m 310$ & $\left\{4,2^{\text {free }}\right\}$ & $0.486(37)$ & & $0.120(19)$ & $8(6)$ & $0.93(10)$ & & & 0.79 \\
\hline$a 06 m 135$ & $\{4,2\}$ & $0.197(37)$ & & $0.0385(97)$ & $0.69(1.75)$ & $2.00(81)$ & & & 1.70 \\
\hline$a 06 m 135$ & $\left\{4^{N \pi}, 2\right\}$ & $0.0846(84)$ & & $\ldots$ & $\ldots$ & $\ldots$ & & & $\ldots$ \\
\hline$a 06 m 135$ & $\left\{4,3^{*}\right\}$ & $0.197(37)$ & $0.287(49)$ & $0.0500(44)$ & $3.6(2.2)$ & $0.61(35)$ & $1.30(43)$ & $-1(6)$ & 1.32 \\
\hline$a 06 m 135$ & $\left\{4^{N \pi}, 3^{*}\right\}$ & $0.0846(84)$ & $0.201(23)$ & $0.082(30)$ & $6(3)$ & $-1.3(8)$ & $1.5(3)$ & $-1.8(1.7)$ & 1.34 \\
\hline$a 06 m 135$ & $\left\{4,2^{\text {free }}\right\}$ & $0.306(81)$ & & $0.0545(62)$ & $17(26)$ & $1.29(14)$ & & & 1.31 \\
\hline
\end{tabular}

\section{CHIRAL, CONTINUUM, AND INFINITE VOLUME EXTRAPOLATION}

To obtain the final, physical results at $M_{\pi}=135 \mathrm{MeV}$, $M_{\pi} L \rightarrow \infty$, and $a=0$, we make a simultaneous CCFV fit keeping only the leading correction term in each variable:

$$
\langle x\rangle\left(M_{\pi} ; a ; L\right)=c_{1}+c_{2} a+c_{3} M_{\pi}^{2}+c_{4} \frac{M_{\pi}^{2} e^{-M_{\pi} L}}{\sqrt{M_{\pi} L}} .
$$

Note that, since the operators are not $O(a)$ improved and we used the Clover-on-HISQ formulation, we take the discretization errors to start with a term linear in $a$. The fits to the $\left\{4,3^{*}\right\}$ data from the nine ensembles are shown in Figs. 1, 2, and 3. The fit parameters are summarized in Table VI.

The results of the CCFV fits show that the finite volume correction term, $c_{4}$, is not constrained. We, therefore, also present results from a CC fit, i.e., with $c_{4}=0$ in Eq. (22). Results for $c_{1}$ from the two fit ansatz overlap, and there is a small positive slope in both $a$ and $M_{\pi}^{2}$ for all three quantities. The data for both $\left\{4,3^{*}\right\}$ and $\left\{4,2^{\text {free }}\right\}$, given in Table II, are very similar, but with a systematic shift of about $0.01-0.02$ in all three cases. This difference arises because $\Delta M_{1}$ for $\left\{4,2^{\text {free }}\right\}$ is larger (except in $a 09 \mathrm{~m} 310$ ) and because the convergence with respect to $\tau$ is from above as shown in Figs. 5 and 6; i.e., a larger $\Delta M_{1}$ implies a smaller extrapolation and a larger $\tau \rightarrow \infty$ value.

For our final results we quote the $\mathrm{CC}$ fit values as the coefficient $c_{4}$ of the finite-volume corrections in the CCFV fits is undetermined. The $\mathrm{CC}$ results with the two strategies, $\left\{4,3^{*}\right\}$ and $\left\{4,2^{\text {free }}\right.$, are summarized in Table VII. For our best estimates, we take the $\left\{4,3^{*}\right\}$ results and add a second, systematic, error that is the difference between these two strategies and represents the uncertainty in controlling the excited-state contamination.
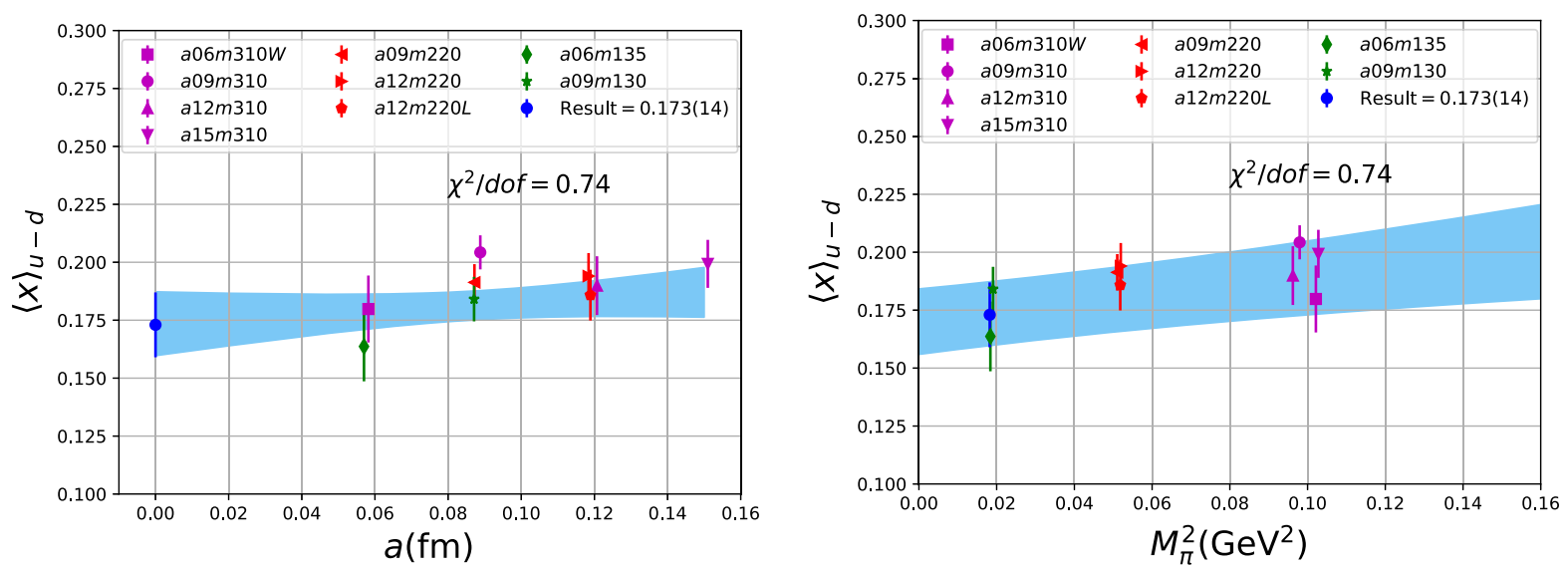

FIG. 1. Data for $\langle x\rangle_{u-d}$, renormalized in the $\overline{\mathrm{MS}}$ scheme at $\mu=2 \mathrm{GeV}$, for all nine ensembles. The blue band in the left panel shows the CC fit result evaluated at $M_{\pi}=135 \mathrm{MeV}$ and plotted versus $a$, while in the right panel it shows the result versus $M_{\pi}^{2}$ evaluated at $a=0$. 

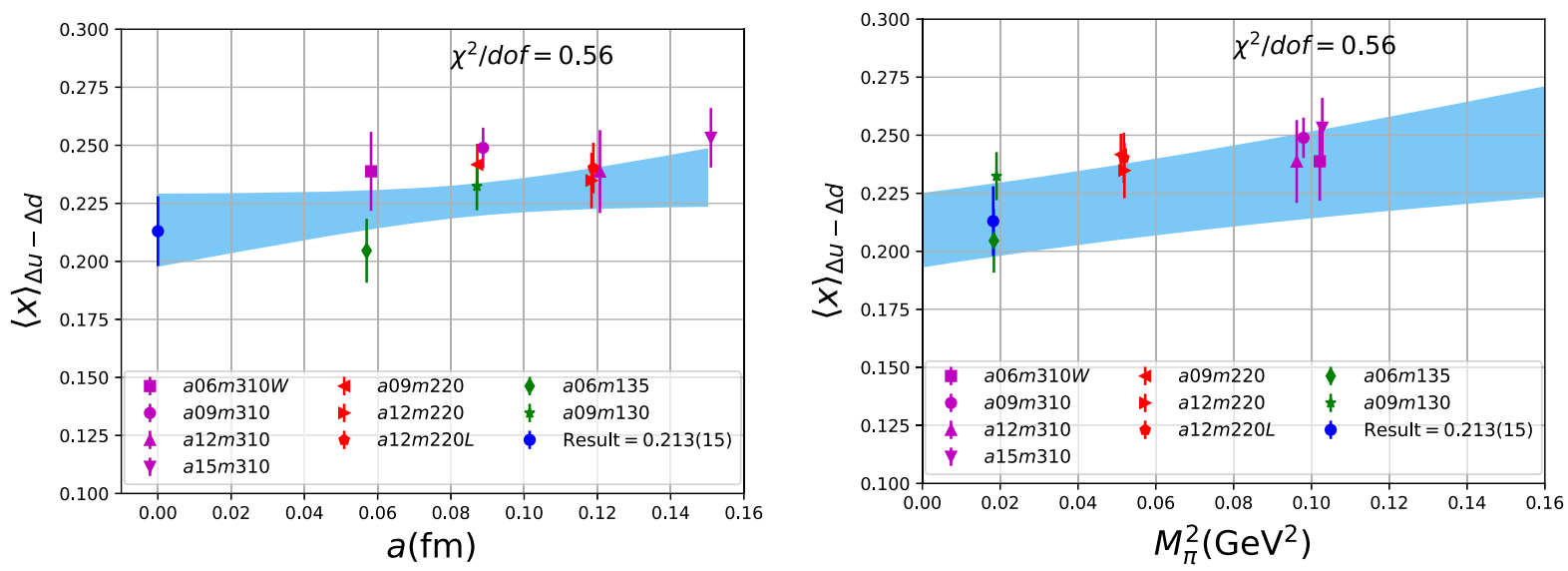

FIG. 2. Data for $\langle x\rangle_{\Delta u-\Delta d}$, renormalized in the $\overline{\mathrm{MS}}$ scheme at $\mu=2 \mathrm{GeV}$, for all nine ensembles plotted as a function of $a$ (left panel) and $M_{\pi}^{2}$ (right panel). The rest is the same as in Fig. 1.
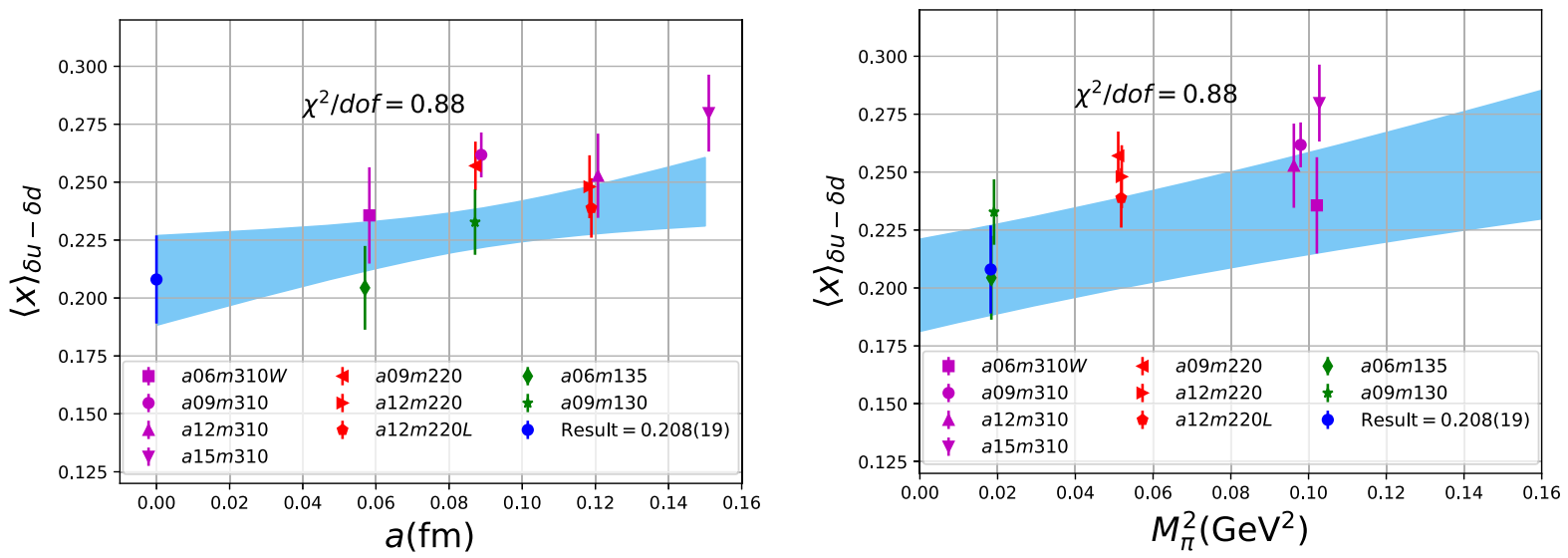

FIG. 3. Data for $\langle x\rangle_{\delta u-\delta d}$, renormalized in the $\overline{\mathrm{MS}}$ scheme at $\mu=2 \mathrm{GeV}$, for all nine ensembles plotted as a function of $a$ (left panel) and $M_{\pi}^{2}$ (right panel). The rest is the same as in Fig. 1.

TABLE VI. Results for the fit parameters in the CCFV ansatz given in Eq. (22) and used for the chiral, continuum, and finite volume (CCFV) extrapolation of the $\left\{4,3^{*}\right\}$ data. The CC and CCFV fit-types correspond to fits with $c_{4}=0$ or $c_{4} \neq 0$.

\begin{tabular}{lcccccc}
\hline \hline Fit-type & Observable & $c_{1}$ & $c_{2}$ & $c_{3}$ & $c_{4}$ & $\chi^{2} /$ dof \\
\hline CC & $\langle x\rangle_{u-d}$ & $0.170(14)$ & $0.09(14)$ & $0.19(11)$ & & 0.74 \\
CC & $\langle x\rangle_{\Delta u-\Delta d}$ & $0.209(16)$ & $0.15(16)$ & $0.24(13)$ & & 0.56 \\
CC & $\langle x\rangle_{\delta u-\delta d}$ & $0.201(20)$ & $0.26(20)$ & $0.35(16)$ & & 0.88 \\
CCFV & $\langle x\rangle_{u-d}$ & $0.167(16)$ & $0.12(16)$ & $0.24(17)$ & $-9(23)$ & 0.85 \\
CCFV & $\langle x\rangle_{\Delta u-\Delta d}$ & $0.206(16)$ & $0.18(17)$ & $0.32(19)$ & $-15(25)$ & 0.59 \\
CCFV & $\langle x\rangle_{\delta u-\delta d}$ & $0.202(21)$ & $0.25(20)$ & $0.34(24)$ & $3(31)$ & 1.06 \\
\hline \hline
\end{tabular}

TABLE VII. Results for the three moments from the two strategies $\left\{4,3^{*}\right\}$ and $\left\{4,2^{\text {free }}\right.$. For our best estimates, we take the $\left\{4,3^{*}\right\}$ values and assign a second, systematic, error that is the difference between the two results. The results are in the $\overline{\mathrm{MS}}$ scheme at scale $2 \mathrm{GeV}$.

\begin{tabular}{lccr}
\hline \hline Observable & $\left\{4,3^{*}\right\}$ & $\left\{4,2^{\text {free }}\right\}$ & Best estimate \\
\hline$\langle x\rangle_{u-d}^{\overline{\mathrm{MS}}}$ & $0.173(14)$ & $0.180(14)$ & $0.173(14)(07)$ \\
$\langle x\rangle_{\Delta u-\Delta d}^{\mathrm{MS}}$ & $0.213(15)$ & $0.235(15)$ & $0.213(15)(22)$ \\
$\langle x\rangle_{\delta u-\delta d}^{\mathrm{MS}}$ & $0.208(19)$ & $0.236(18)$ & $0.208(19)(24)$ \\
\hline \hline
\end{tabular}



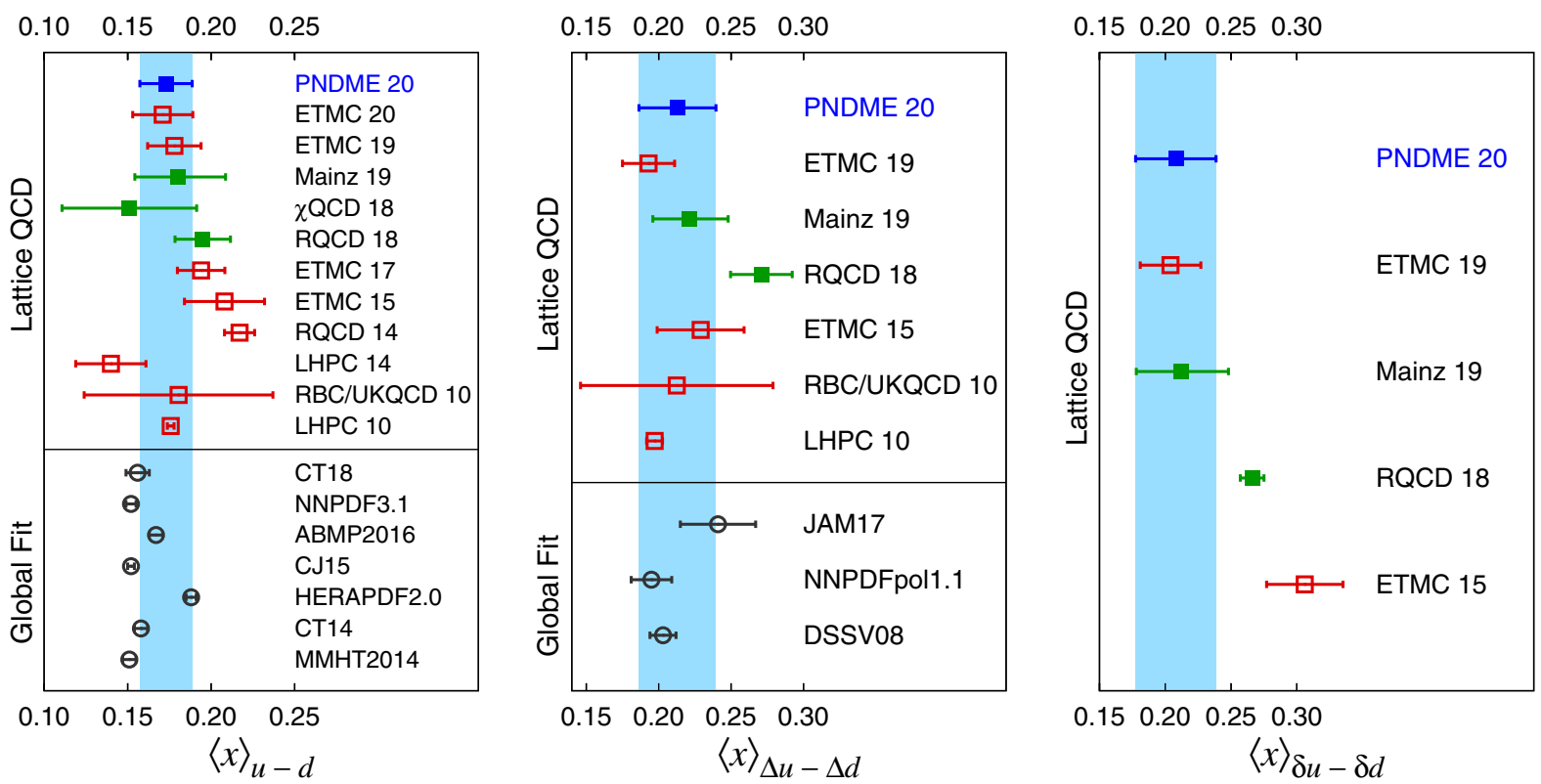

FIG. 4. A comparison of results from lattice QCD calculations with dynamical fermions and global fits (below the black line) summarized in Table VIII. The left panel compares results for the momentum fraction, the middle for the helicity moment, and the right for the transversity moment. The PNDME 20 result is also shown as the blue band to facilitate comparison.

A comparison of these results with other lattice QCD calculations on ensembles with dynamical fermions is presented in the top half of Table VIII and shown in Fig. 4. Our results agree with those from the Mainz group [20] that have also been obtained using data on a comparable number of ensembles, but all with $M_{\pi}>200 \mathrm{MeV}$, which are used to perform a chiral and continuum extrapolation. The one difference is the slope $c_{3}$ of the chiral correction. For our clover-onHISQ formulation, we find a small positive value while the Mainz data show a small negative value [20]. Our results are also consistent within $1 \sigma$ with the ETMC 20 [32] and ETMC 19 [33] values that are from a single physical mass ensemble. The central value from $\chi \mathrm{QCD}$ [34], using partially quenched analysis, is smaller but consistent within $1 \sigma$. Results for the momentum fraction and the helicity moment from RQCD 18 [35] are taken from their Set A with the difference between Set A and B values quoted as a second systematic uncertainty. Their result for the transversity moment is from a single $150 \mathrm{MeV}$ ensemble. These values are larger, especially for the helicity and transversity moment. Other earlier lattice results show a spread; however, in each of these calculations, the systematics listed in the last column of Table VIII have not been addressed or controlled and could, therefore, account for the differences.

Estimates from phenomenological global fits, most of which have also been reviewed in Ref. [6], are summarized in the bottom of Table VIII and shown in Fig. 4. We find that results for the momentum fraction from global fits are, in most cases, $1-2 \sigma$ smaller and have much smaller errors. Results for the helicity moment are consistent and the size of the errors comparable. Lattice estimates of the transversity moment are a prediction.

\section{CONCLUSIONS}

In this paper, we have presented results for the isovector quark momentum fraction, $\langle x\rangle_{u-d}^{\overline{\mathrm{MS}}}$, helicity moment, $\langle x\rangle_{\Delta u-\Delta d}^{\overline{\mathrm{MS}}}$, and transversity moment, $\langle x\rangle_{\delta u-\delta d}^{\overline{\mathrm{MS}}}$, from a high statistics lattice QCD calculation. Attention has been paid to the systematic uncertainty associated with excited-state contamination. We have carried out the full analysis with different estimates of the mass gaps of possible excited states, and we use the difference in results between the two strategies that give stable fits on all ensembles as an additional systematic uncertainty to account for possible residual excited-state contamination.

The behavior versus $M_{\pi}$, the lattice spacing $a$, and finite volume parameter $M_{\pi} L$ have been investigated using a simultaneous fit that includes the leading correction in all three variables as given in Eq. (22). The nine data points cover the range $0.057<a<0.15 \mathrm{fm}$, $135<M_{\pi}<320 \mathrm{MeV}$, and $3.7<M_{\pi} L<5.5$. Over this range, all three moments, $\langle x\rangle_{u-d}^{\overline{\mathrm{MS}}},\langle x\rangle_{\Delta u-\Delta d}^{\overline{\mathrm{MS}}}$, and $\langle x\rangle_{\delta u-\delta d}^{\overline{\mathrm{MS}}}$, do not show a large dependence on $a$ or $M_{\pi}$ or $M_{\pi} L$. As shown in Table VI, possible dependence on the lattice size, characterized by $M_{\pi} L$, is not resolved by the data; i.e., the coefficient $c_{4}$ is unconstrained. We, therefore, take for our final results those obtained from just the chiralcontinuum fit. 
TABLE VIII. Our lattice QCD results are compared with other lattice calculations with $N_{f}$ flavors of dynamical fermions in rows $2-$ 12 , and with results from phenomenological global fits in the remainder of the table. In both cases, the results are arranged in reverse chronological order. All results are in the $\overline{\mathrm{MS}}$ scheme at scale $2 \mathrm{GeV}$. For a discussion and comparison of lattice and global fit results up to 2017 see Ref. [6]; and for a more recent comparison of $\langle x\rangle_{u-d}$ see Ref. [41]. The JAM17 ${ }^{\dagger}$ estimate for $\langle x\rangle_{\Delta u-\Delta d}$ is obtained from [6], where, as part of the review, an analysis was carried out using the data in [37]. The following abbreviations are used in the remarks column for various sources of systematic uncertainties in lattice calculations-DIS: discretization effects; CE: chiral extrapolation; FV: finite volume effects; NR: nonperturbative renormalization; ES: excited state contaminations. A prefix "N-" means that the systematic uncertainty was not adequately controlled or not estimated.

\begin{tabular}{|c|c|c|c|c|c|}
\hline Collaboration & Ref. & $\langle x\rangle_{u-d}$ & $\langle x\rangle_{\Delta u-\Delta d}$ & $\langle x\rangle_{\delta u-\delta d}$ & Remarks \\
\hline $\begin{array}{l}\text { PNDME } 20 \\
\text { (this work) }\end{array}$ & & $0.173(14)(07)$ & $0.213(15)(22)$ & $0.208(19)(24)$ & $N_{f}=2+1+1$ clover-on-HISQ \\
\hline ETMC 20 & {$[32]$} & $0.171(18)$ & & & $N_{f}=2+1+1$ twisted mass N-DIS, N-FV \\
\hline ETMC 19 & {$[33]$} & $0.178(16)$ & $0.193(18)$ & $0.204(23)$ & $N_{f}=2+1+1$ twisted mass N-DIS, N-FV \\
\hline Mainz 19 & {$[20]$} & $\begin{array}{l}0.180(25)_{\text {stat }} \\
(+14,-6)_{\text {sys }}\end{array}$ & $\begin{array}{l}0.221(25)_{\text {stat }} \\
(+10,-0)_{\text {sys }}\end{array}$ & $\begin{array}{l}0.212(32)_{\text {stat }} \\
(+16,-10)_{\text {sys }}\end{array}$ & $N_{f}=2+1$ clover \\
\hline$\chi \mathrm{QCD} 18$ & {$[34]$} & $0.151(28)(29)$ & & & $N_{f}=2+1$ overlap on domain wall \\
\hline RQCD 18 & [35] & $0.195(07)(15)$ & $0.271(14)(16)$ & $0.266(08)(04)$ & $N_{f}=2$ clover \\
\hline ETMC 17 & {$[38]$} & $0.194(9)(11)$ & & & $N_{f}=2$ twisted mass N-DIS, N-FV \\
\hline ETMC 15 & [39] & $0.208(24)$ & $0.229(30)$ & $0.306(29)$ & $N_{f}=2$ twisted mass N-DIS, N-FV \\
\hline RQCD 14 & {$[25]$} & $0.217(9)$ & & & $N_{f}=2$ clover N-DIS, N-CE, N-FV \\
\hline LHPC 14 & {$[40]$} & $0.140(21)$ & & & $N_{f}=2+1$ clover N-DIS $(a \sim 0.12 \mathrm{fm})$ \\
\hline RBC/UKQCD 10 & [41] & $0.124-0.237$ & $0.146-0.279$ & & $N_{f}=2+1$ domain wall N-DIS, N-CE, N-ES \\
\hline LHPC 10 & {$[42]$} & $0.1758(20)$ & $0.1972(55)$ & & $\begin{array}{c}N_{f}=2+1 \text { domain wall-on-asqtad } \\
\text { N-DIS, N-CE, N-NR, N-ES }\end{array}$ \\
\hline CT18 & [36] & $0.156(7)$ & & & \\
\hline JAM17 $7^{\dagger}$ & {$[6,37]$} & & $0.241(26)$ & & \\
\hline NNPDF3.1 & {$[43]$} & $0.152(3)$ & & & \\
\hline ABMP2016 & [44] & $0.167(4)$ & & & \\
\hline CJ15 & {$[45]$} & $0.152(2)$ & & & \\
\hline HERAPDF2.0 & [46] & $0.188(3)$ & & & \\
\hline CT14 & [47] & $0.158(4)$ & & & \\
\hline МMHT2014 & [48] & $0.151(4)$ & & & \\
\hline NNPDFpol1.1 & [49] & & $0.195(14)$ & & \\
\hline DSSV08 & {$[50,51]$} & & $0.203(9)$ & & \\
\hline
\end{tabular}

The small increase with $a$ and $M_{\pi}^{2}$, evident in Figs. 1-3, is well fit by the leading correction terms that are linear in these variables. Also, for all three observables, the chirally extrapolated value is consistent with the data from the two physical mass ensembles. In short, the observed small dependence in all three variables, and having two data points at $M_{\pi} \sim 135 \mathrm{MeV}$ to anchor the chiral fit, allows a controlled extrapolation to the physical point, $M_{\pi}=$ $135 \mathrm{MeV}$ and $a=0$.

Our final results, given in Table VII, are compared with other lattice calculations and phenomenological global fit estimates in Table VIII and shown in Fig. 4. Estimates of all three quantities are in good agreement with those from the Mainz Collaboration [20], also obtained using a chiralcontinuum extrapolation, from the ETMC Collaboration $[32,33]$ that are from a single physical mass ensemble, and from the $\chi \mathrm{QCD}$ Collaboration [34]. On the other hand, most global fit estimates for the momentum fraction are about $10 \%$ smaller and have much smaller errors, while those for the helicity moment are consistent within $1 \sigma$. Lattice estimates for the transversity moment are a prediction. The overall consistency of results suggests that lattice QCD calculations of these isovector moments are now mature and future calculations will steadily reduce the statistical and systematic uncertainties in them.

\section{ACKNOWLEDGMENTS}

We thank the MILC Collaboration for sharing the HISQ ensembles, and Martha Constantinou, Giannis Koutsou, Emanuele Nocera, and Juan Rojo for discussions. The calculations used the Chroma software suite [52]. 
Simulations were carried out on computer facilities of (i) the National Energy Research Scientific Computing Center, a DOE Office of Science User Facility supported by the Office of Science of the U.S. Department of Energy under Contract No. DE-AC02-05CH11231; (ii) the Oak
Ridge Leadership Computing Facility at the Oak Ridge National Laboratory, which is supported by the Office of Science of the U.S. Department of Energy under Contract No. DE-AC05-00OR22725; (iii) the USQCD Collaboration, which is funded by the Office of Science
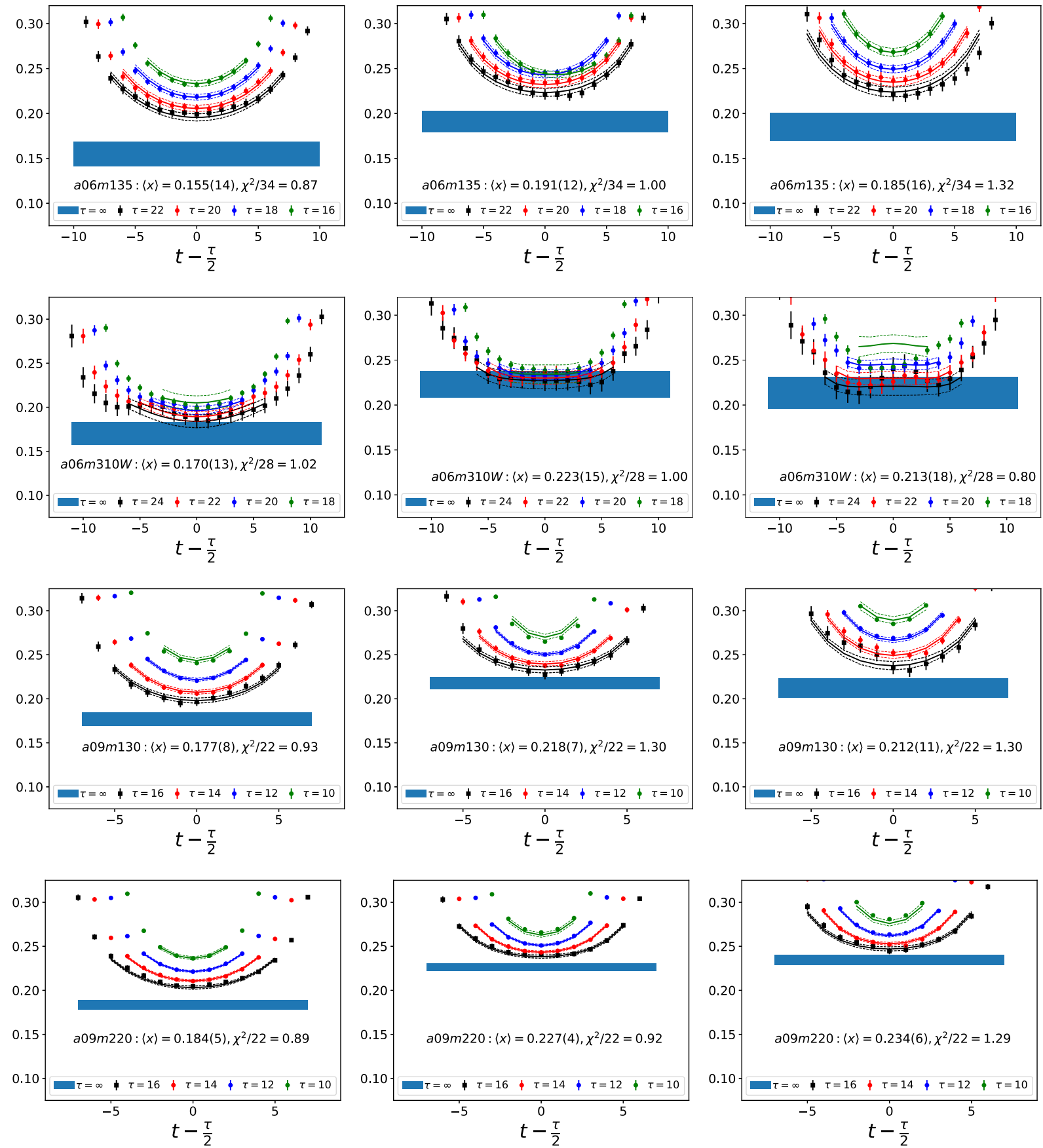

FIG. 5. Data and fits for $a 06 m 135$ (top row), $a 06 m 310 W$ (second row), $a 09 m 130$ (third row), and $a 09 m 220$ (last row). In each row, the three panels show the ratio $C_{\mathcal{O}}^{3 \mathrm{pt}}(\tau ; t) / C^{2 \mathrm{pt}}(\tau)$ scaled according to Eq. (17)-(19) to give $\langle x\rangle_{u-d}$ (left), $\langle x\rangle_{\Delta u-\Delta d}$ (middle), and $\langle x\rangle_{\delta u-\delta d}$ (right). For each $\tau$, the line in the same color as the data points is the result of the $\left\{4,3^{*}\right\}$ fit (see Sec. V) used to obtain the ground state matrix element. The ensemble ID, the final result $\langle x\rangle$ (also shown by the blue band and summarized in Table II), the values of $\tau$, and $\chi^{2} /$ dof of the fit are also given in the legends. The interval of the $y$ axis is selected to be the same for all the panels to facilitate comparison. 

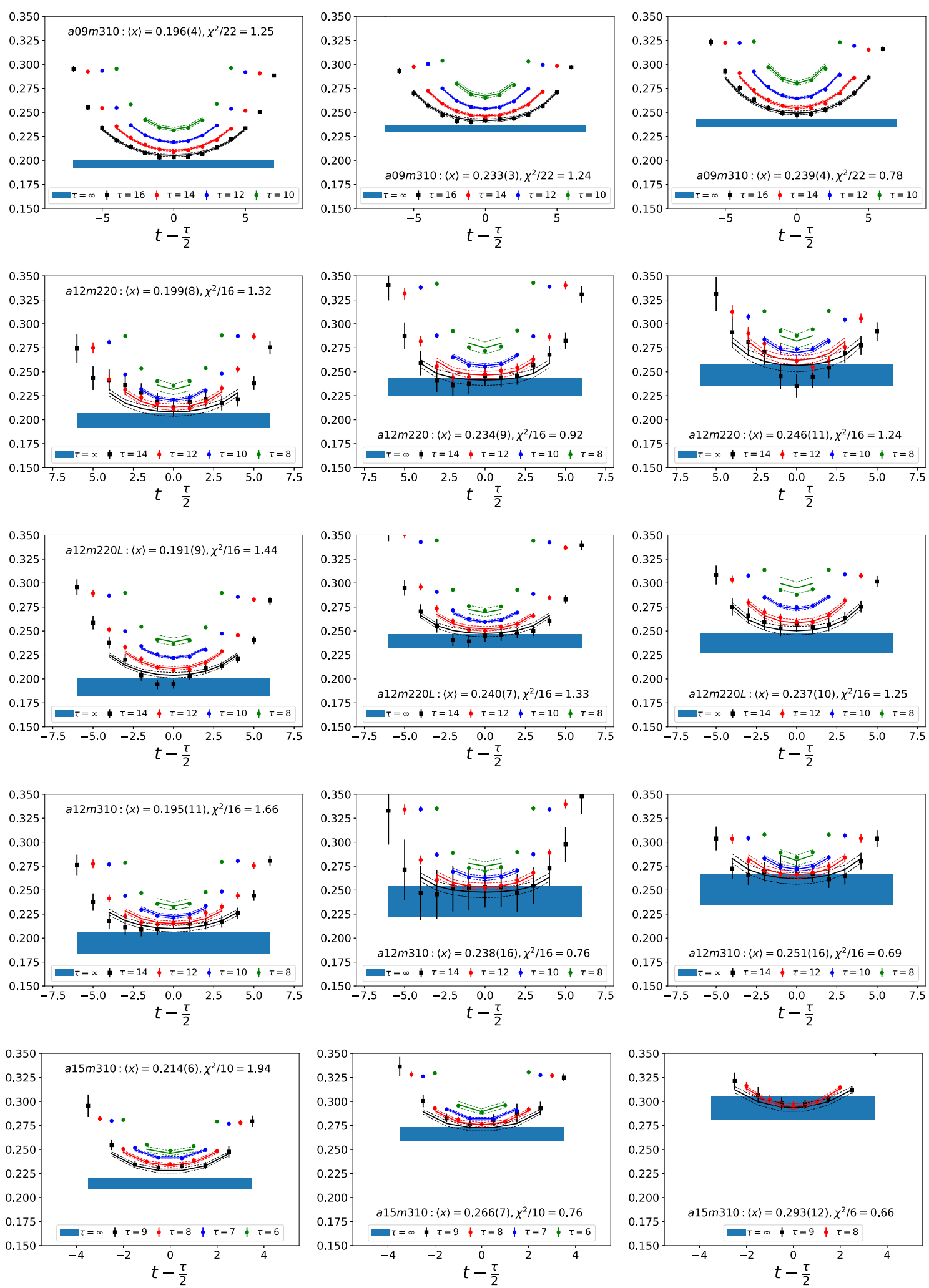

FIG. 6. Data and fits for $a 09 m 310$ (top row), $a 12 m 220$ (second row), $a 12 m 220 L$ (third row), $a 12 m 310$ (fourth row), and $a 15 m 310$ (bottom row) ensembles. The rest is the same as in Fig. 5. 
of the U.S. Department of Energy; and (iv) Institutional Computing at Los Alamos National Laboratory. T. B. and R. G. were partly supported by the U.S. Department of Energy, Office of Science, Office of High Energy Physics under Contract No. DE-AC52-06NA25396. T. B., R. G., S. M., S. P., and B. Y. were partly supported by the LANL LDRD program, and S.P. by the Center for Nonlinear Studies. The work of H.-W. Lin is partly supported by the U.S. National Science Foundation under Grant No. PHY 1653405 "CAREER: Constraining Parton Distribution Functions for New-Physics Searches" and by the Research Corporation for Science Advancement through the Cottrell Scholar Award.

\section{APPENDIX A: PLOTS OF THE RATIO $C_{\mathcal{O}}^{3 p t}(\tau ; t) / C^{2 p t}(\tau)$}

In this Appendix, we show in Figs. 5 and 6, plots of the unrenormalized isovector momentum fraction, $\langle x\rangle_{u-d}$, the helicity moment, $\langle x\rangle_{\Delta u-\Delta d}$, and the transversity moment, $\langle x\rangle_{\delta u-\delta d}$, for the nine ensembles. The data show the ratio $C_{\mathcal{O}}^{3 \mathrm{pt}}(\tau ; t) / C^{2 \mathrm{pt}}(\tau)$ multiplied by the appropriate factor given in Eqs. (17)-(19) to get $\langle x\rangle$. The lines with the same color as the data are the result of the fit to $C_{\mathcal{O}}^{3 \mathrm{pt}}(\tau ; t)$ using Eq. (21). In all cases, to extract the ground state matrix element, the fits to $C^{2 \mathrm{pt}}(\tau)$ and $C_{\mathcal{O}}^{3 \mathrm{pt}}(\tau ; t)$ are done within a single jackknife loop.

\section{APPENDIX B: RENORMALIZATION}

In this Appendix, we describe the calculation of the renormalization factors, $Z_{V D, A D, T D}$, for the three onederivative operators. These are determined nonperturbatively on the lattice in the $\mathrm{RI}^{\prime}-\mathrm{MOM}$ scheme $[53,54]$ as a function of the lattice scale $p^{2}=p^{\mu} p^{\mu}$, and then converted to the $\overline{\mathrm{MS}}$ scheme using 3-loop perturbative factors calculated in the continuum in Ref. [55]. For data at each $p$, we perform horizontal matching by choosing the $\overline{\mathrm{MS}}$ scale $\mu=|p|$. These numbers are then run in the continuum
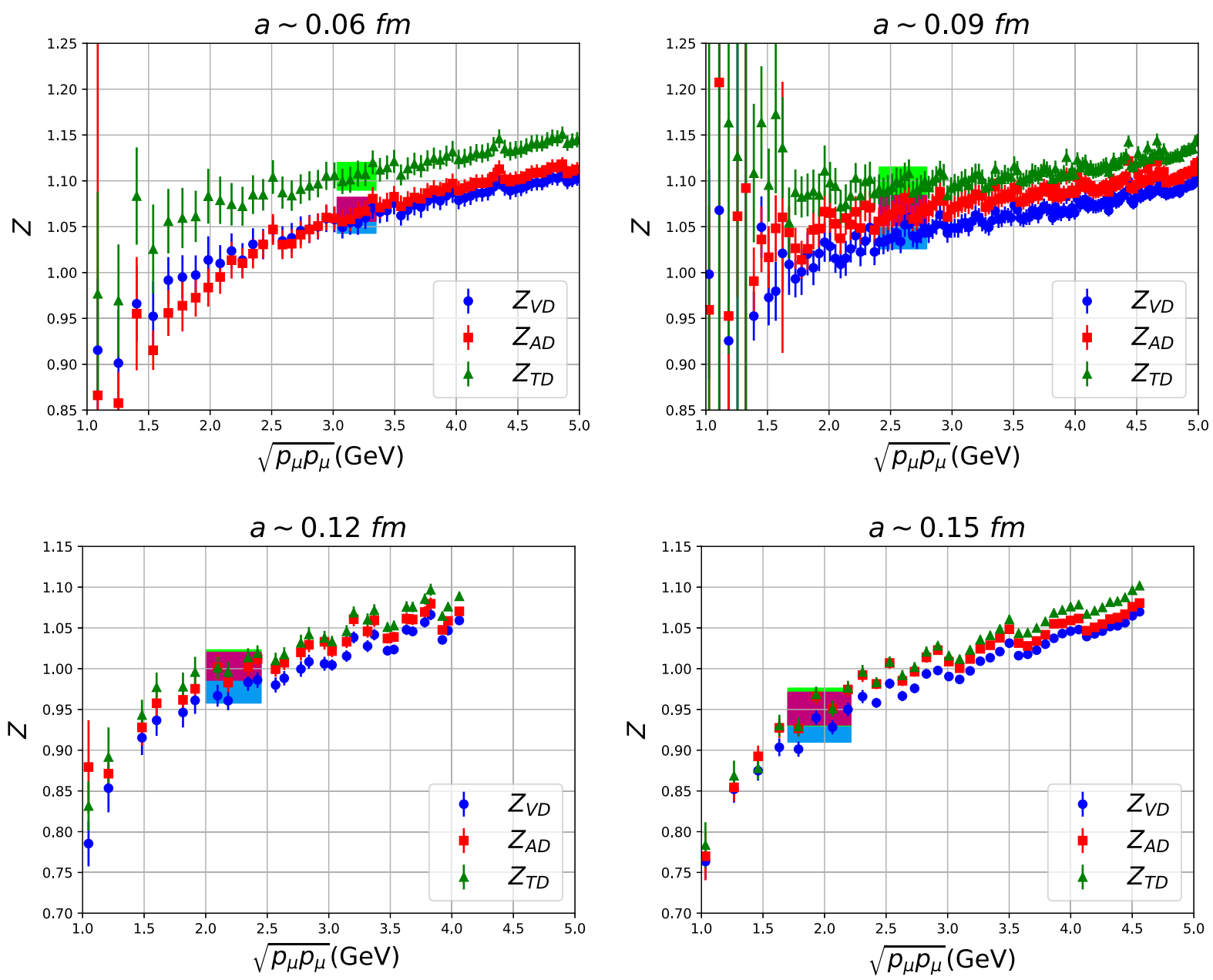

FIG. 7. Nonperturbative renormalization factors for $\langle x\rangle_{u-d},\left(Z_{V D}\right),\langle x\rangle_{\Delta u-\Delta d},\left(Z_{A D}\right)$, and $\langle x\rangle_{\delta u-\delta d},\left(Z_{T D}\right)$, at the four lattice spacings in the $\overline{\mathrm{MS}}$ scheme at $\mu=2 \mathrm{GeV}$. The shaded bands mark the region in $\sqrt{p^{2}}$ that is averaged and the error in the estimate. 


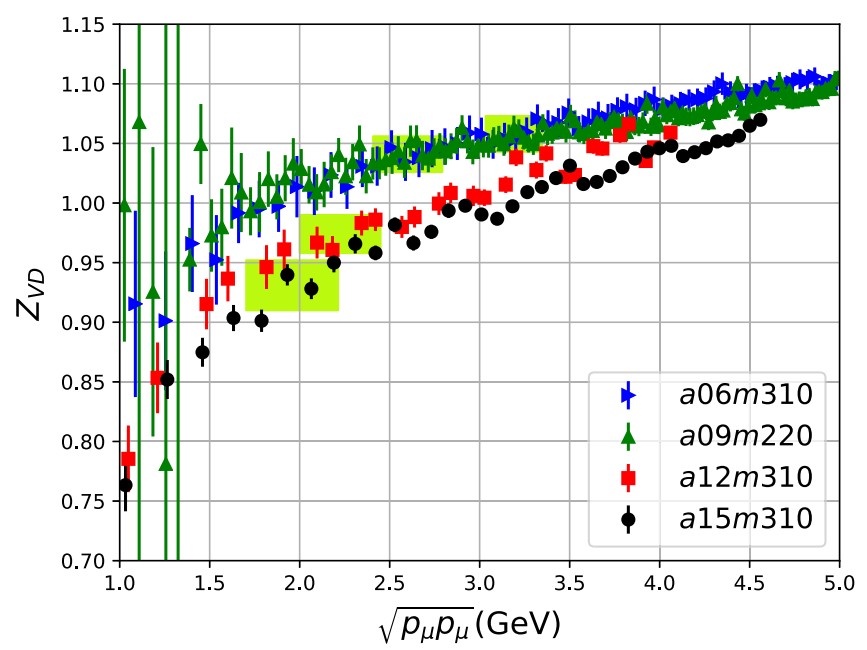

FIG. 8. Nonperturbative renormalization factor $Z_{V D}$ for $\langle x\rangle_{u-d}$ is calculated on four ensembles, one from each lattice spacing. The shaded bands give the interval in $\sqrt{p^{2}}$ over which the data are averaged to get the result and the error in the estimate.

$\overline{\mathrm{MS}}$ scheme from scale $\mu$ to $2 \mathrm{GeV}$ using three-loop anomalous dimensions [55]. Note that the decomposition of the three operators into irreducible representations given in Refs. $[19,20]$ shows that they can only mix with higher dimensional operators. Such $O(a)$ effects would also be taken into account in our CCFV fits, and removed by the continuum extrapolation.

We calculate $Z_{V D, A D, T D}$ for one value of $M_{\pi}$ at each $a$. Based on our experience with local operators [13], where we found insignificant dependence of results on $M_{\pi}$, we assume that these results, within the conservative error estimates we assign, give the mass-independent renormalization factors at each $a$. Evidence that the dependence on $M_{\pi}^{2}$ is tiny for these 1-link operators also comes from explicit calculations in Refs. [20,32], albeit with different lattice actions. In each case, the dependence on $M_{\pi}^{2}$ is found to be much smaller than $1 \%$. The dominant uncertainty comes from the dependence on $p^{2}$, which is discussed next.

In Fig. 7, we show the behavior of the renormalization factors $Z_{V D, A D, T D}$ in the $\overline{\mathrm{MS}}$ scheme at $\mu=2 \mathrm{GeV}$ for the four ensembles as a function of $|p|$-the scale of the $\mathrm{RI}^{\prime}$-MOM scheme on the lattice. In Fig. 8 we compare $Z_{V D}$,
TABLE IX. Results for the renormalization factors, $Z_{V D, A D, T D}$, in the $\overline{\mathrm{MS}}$ scheme at $2 \mathrm{GeV}$. These are calculated in the $\mathrm{RI}^{\prime}-\mathrm{MOM}$ scheme as a function of scale $p=\sqrt{p_{\mu} p_{\mu}}$ on the lattice, matched to the $\overline{\mathrm{MS}}$ scheme at the same scale $\mu=p$, and then run in the continuum $\overline{\mathrm{MS}}$ scheme from $\mu$ to $2 \mathrm{GeV}$. The results are the average of values over the range of $|p|$ specified in the second column. The final error estimate is taken to be twice that shown in Figs. 7 and 8 .

\begin{tabular}{lcccc}
\hline \hline$a(\mathrm{fm})$ & $\begin{array}{c}\text { Fit range } \\
{\left[\mathrm{GeV}^{2}\right]}\end{array}$ & $Z_{V D}$ & $Z_{A D}$ & $Z_{T D}$ \\
\hline 0.06 & $9.2-11.2$ & $1.058(30)$ & $1.069(26)$ & $1.105(30)$ \\
0.09 & $5.8-7.8$ & $1.041(30)$ & $1.067(34)$ & $1.097(36)$ \\
0.12 & $4.0-6.0$ & $0.974(32)$ & $1.003(34)$ & $1.007(32)$ \\
0.15 & $2.9-4.9$ & $0.931(42)$ & $0.951(40)$ & $0.953(46)$ \\
\hline \hline
\end{tabular}

used to renormalize $\langle x\rangle_{u-d}$, calculated on four ensembles, one at each lattice spacing.

For all three operators, the data do not show a window in $|p|$ where the results are independent of $|p|$. The variation in the data is due to a combination of the breaking of full rotational invariance on the lattice and other $p^{2}$ dependent artifacts. This is the dominant uncertainty and many methods have been proposed to control it; see for example Refs. [13,20,32]. In Ref. [13], we explored three methods that gave consistent results, and of these we have selected the strategy labeled "Method B" there as it is the most straightforward. In this approach, we take an average over the data points in an interval of $2 \mathrm{GeV}^{2}$ about $p^{2}=\Lambda / a$, where the scale $\Lambda=3 \mathrm{GeV}$ is chosen to be large enough to avoid nonperturbative effects and at which perturbation theory is expected to be reasonably well behaved. Also, this choice satisfies both $p a \rightarrow 0$ and $\Lambda / p \rightarrow 0$ in the continuum limit as desired. The window over which the data are averaged and the error (half the height of the band) are shown by shaded bands in Figs. 7 and 8. Noting the large variation with $p^{2}$, we take twice this error, i.e., full height of the band, for a very conservative error estimate for all three $Z^{\prime}$ s.

These final estimates of $Z_{V D}, Z_{A D}$, and $Z_{T D}$ used to renormalize the momentum fraction, the helicity moment, and the transversity moment, respectively, are given in Table IX.
[1] R. Brock et al. (CTEQ Collaboration), Rev. Mod. Phys. 67, 157 (1995).

[2] B. Yoon, M. Engelhardt, R. Gupta, T. Bhattacharya, J. R. Green, B. U. Musch, J. W. Negele, A. V. Pochinsky, A. Schafer, and S. N. Syritsyn, Phys. Rev. D 96, 094508 (2017).
[3] M. Diehl, Phys. Rep. 388, 41 (2003).

[4] A. Accardi et al., Eur. Phys. J. A 52, 268 (2016).

[5] D. Boer et al., arXiv:1108.1713.

[6] H.-W. Lin et al., Prog. Part. Nucl. Phys. 100, 107 (2018).

[7] S. Aoki et al. (Flavour Lattice Averaging Group), Eur. Phys. J. C 80, 113 (2020). 
[8] K. Cichy and M. Constantinou, Adv. High Energy Phys. 2019, 3036904 (2019).

[9] N. Karthik, Lattice computations of PDF: Challenges and progress, https://indico.cern.ch/event/764552/contributions/ 3420535/attachments/1864018/3064443/LatticeTalk19_ NK.pdf (2019), accessed: 2020-05-20.

[10] E. Follana, Q. Mason, C. Davies, K. Hornbostel, G. P. Lepage, J. Shigemitsu, H. Trottier, and K. Wong (HPQCD Collaboration, UKQCD Collaboration), Phys. Rev. D 75, 054502 (2007).

[11] A. Bazavov et al. (MILC Collaboration), Phys. Rev. D 87, 054505 (2013).

[12] T. Bhattacharya, V. Cirigliano, S. Cohen, R. Gupta, A. Joseph, H.-W. Lin, and B. Yoon (PNDME Collaboration), Phys. Rev. D 92, 094511 (2015).

[13] T. Bhattacharya, V. Cirigliano, S. Cohen, R. Gupta, H.-W. Lin, and B. Yoon, Phys. Rev. D 94, 054508 (2016).

[14] R. Gupta, Y.-C. Jang, B. Yoon, H.-W. Lin, V. Cirigliano, and T. Bhattacharya, Phys. Rev. D 98, 034503 (2018).

[15] B. Sheikholeslami and R. Wohlert, Nucl. Phys. B259, 572 (1985).

[16] A. Hasenfratz and F. Knechtli, Phys. Rev. D 64, 034504 (2001).

[17] G. S. Bali, S. Collins, and A. Schafer, Comput. Phys. Commun. 181, 1570 (2010).

[18] T. Blum, T. Izubuchi, and E. Shintani, Phys. Rev. D 88, 094503 (2013).

[19] M. Gockeler, R. Horsley, E.-M. Ilgenfritz, H. Perlt, P. E. L. Rakow, G. Schierholz, and A. Schiller, Phys. Rev. D 53, 2317 (1996).

[20] T. Harris, G. von Hippel, P. Junnarkar, H. B. Meyer, K. Ottnad, J. Wilhelm, H. Wittig, and L. Wrang, Phys. Rev. D 100, 034513 (2019).

[21] Z. Davoudi and M. J. Savage, Phys. Rev. D 86, 054505 (2012).

[22] W. Detmold and C. J. D. Lin, Phys. Rev. D 73, 014501 (2006).

[23] W. Detmold, I. Kanamori, C. J. D. Lin, S. Mondal, and Y. Zhao, Proc. Sci., LATTICE2018 (2018) 106 [arXiv:1810.12194].

[24] T. Bhattacharya, S. D. Cohen, R. Gupta, A. Joseph, H.-W. Lin, and B. Yoon, Phys. Rev. D 89, 094502 (2014).

[25] G. S. Bali, S. Collins, B. Glle, M. Gckeler, J. Najjar, R. H. Rdl, A. Schfer, R. W. Schiel, A. Sternbeck, and W. Sldner, Phys. Rev. D 90, 074510 (2014).

[26] G. S. Bali, S. Collins, M. Deka, B. Glassle, M. Gockeler, J. Najjar, A. Nobile, D. Pleiter, A. Schafer, and A. Sternbeck, Phys. Rev. D 86, 054504 (2012).

[27] B. Yoon et al., Phys. Rev. D 93, 114506 (2016).

[28] R. Babich, J. Brannick, R. Brower, M. Clark, T. Manteuffel, S. F. McCormick, J. C. Osborn, and C. Rebbi, Phys. Rev. Lett. 105, 201602 (2010).

[29] M. Clark, R. Babich, K. Barros, R. Brower, and C. Rebbi, Comput. Phys. Commun. 181, 1517 (2010).

[30] B. Yoon et al., Phys. Rev. D 95, 074508 (2017).
[31] Y.-C. Jang, R. Gupta, B. Yoon, and T. Bhattacharya, Phys. Rev. Lett. 124, 072002 (2020).

[32] C. Alexandrou, S. Bacchio, M. Constantinou, J. Finkenrath, K. Hadjiyiannakou, K. Jansen, G. Koutsou, H. Panagopoulos, and G. Spanoudes, Phys. Rev. D 101, 094513 (2020).

[33] C. Alexandrou et al., Phys. Rev. D 101, 034519 (2020).

[34] Y.-B. Yang, J. Liang, Y.-J. Bi, Y. Chen, T. Draper, K.-F. Liu, and Z. Liu, Phys. Rev. Lett. 121, 212001 (2018).

[35] G. S. Bali, S. Collins, M. Göckeler, R. Rödl, A. Schfer, and A. Sternbeck, Phys. Rev. D 100, 014507 (2019).

[36] T.-J. Hou et al., arXiv:1912.10053.

[37] J. Ethier, N. Sato, and W. Melnitchouk, Phys. Rev. Lett. 119, 132001 (2017).

[38] C. Alexandrou, M. Constantinou, K. Hadjiyiannakou, K. Jansen, C. Kallidonis, G. Koutsou, A. Vaquero Avils-Casco, and C. Wiese, Phys. Rev. Lett. 119, 142002 (2017).

[39] A. Abdel-Rehim et al., Phys. Rev. D 92, 114513 (2015); 93, 039904(E) (2016).

[40] J. R. Green, M. Engelhardt, S. Krieg, J. W. Negele, A. V. Pochinsky, and S. N. Syritsyn, Phys. Lett. B 734, 290 (2014).

[41] Y. Aoki, T. Blum, H.-W. Lin, S. Ohta, S. Sasaki, R. Tweedie, J. Zanotti, and T. Yamazaki, Phys. Rev. D 82, 014501 (2010).

[42] J. Bratt et al. (LHPC Collaboration), Phys. Rev. D 82, 094502 (2010).

[43] R. D. Ball et al. (NNPDF Collaboration), Eur. Phys. J. C 77, 663 (2017).

[44] S. Alekhin, J. Blmlein, S. Moch, and R. Placakyte, Phys. Rev. D 96, 014011 (2017).

[45] A. Accardi, L. T. Brady, W. Melnitchouk, J. F. Owens, and N. Sato, Phys. Rev. D 93, 114017 (2016).

[46] H. Abramowicz et al. (H1, ZEUS Collaborations), Eur. Phys. J. C 75, 580 (2015).

[47] S. Dulat, T.-J. Hou, J. Gao, M. Guzzi, J. Huston, P. Nadolsky, J. Pumplin, C. Schmidt, D. Stump, and C. P. Yuan, Phys. Rev. D 93, 033006 (2016).

[48] L. A. Harland-Lang, A. D. Martin, P. Motylinski, and R. S. Thorne, Eur. Phys. J. C 75, 204 (2015).

[49] E. R. Nocera, R. D. Ball, S. Forte, G. Ridolfi, and J. Rojo (NNPDF Collaboration), Nucl. Phys. B887, 276 (2014).

[50] D. de Florian, R. Sassot, M. Stratmann, and W. Vogelsang, Phys. Rev. D 80, 034030 (2009).

[51] D. de Florian, R. Sassot, M. Stratmann, and W. Vogelsang, Phys. Rev. Lett. 101, 072001 (2008).

[52] R. G. Edwards and B. Joo (SciDAC, LHPC, UKQCD Collaborations), Nucl. Phys. B Proc. Suppl. 140, 832 (2005).

[53] M. Gockeler et al., Phys. Rev. D 82, 114511 (2010); 86, 099903(E) (2012).

[54] M. Constantinou, M. Costa, M. Gckeler, R. Horsley, H. Panagopoulos, H. Perlt, P. E. L. Rakow, G. Schierholz, and A. Schiller, Phys. Rev. D 87, 096019 (2013).

[55] J. A. Gracey, Nucl. Phys. B667, 242 (2003). 